\title{
An integrative in silico methodology for the identification of modulators of macrophage migration inhibitory factor (MIF) tautomerase activity
}

\author{
Farah El Turk ${ }^{\mathrm{a}, \dagger}$, Bruno Fauvet ${ }^{\mathrm{a}, \dagger}$, Hajer Ouertatani-Sakouhi ${ }^{\mathrm{a}}$, Adrien Lugari ${ }^{\mathrm{b}}$, Stephane Betzi ${ }^{\mathrm{b}}$, \\ Philippe Roche ${ }^{\mathrm{b}}$, Xavier Morelli ${ }^{\mathrm{b}, *}$, Hilal A. Lashuel ${ }^{\mathrm{a}, *}$ \\ a Laboratory of Molecular Neurobiology and Neuroproteomics, Brain Mind Institute, Ecole Polytechnique Fédérale de Lausanne (EPFL), CH-1015 Lausanne, Switzerland

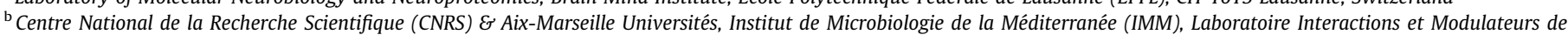 \\ Réponses (UPR3243), 31 Chemin Joseph Aiguier, 13402 Marseille Cedex 20, France
}

\section{A R T I C L E I N F O}

Article history:

Received 9 March 2010

Revised 29 April 2010

Accepted 4 May 2010

Available online 13 May 2010

\begin{abstract}
A B S T R A C T
Macrophage migration inhibitory factor (MIF) is a major proinflammatory cytokine that has been increasingly implicated in the pathogenesis of several inflammatory, autoimmune, infectious and oncogenic diseases. Accumulating evidence suggests that the tautomerase activity of MIF plays a role in modulating some of its intra- and extra-cellular activities. Therefore, the identification and development of smallmolecule inhibitors targeting the catalytic activity of MIF has emerged as an attractive and viable therapeutic strategy to attenuate its function in health and disease. Herein we report a novel virtual screening protocol for the discovery of new inhibitors of MIF's tautomerase activity. Our protocol takes into account the flexibility and dynamics of the catalytic site by coupling molecular dynamics (MD) simulations aimed at modeling the protein's flexibility in solution to (i) docking with FlexX, or (ii) docking with FlexX and pharmacophoric filtering with Unity. In addition, we applied in parallel a standalone docking using the new version of Surflex software. The three approaches were used to screen the ChemBridge chemical library and the inhibitory activity of the top-ranked 333 compound obtained from each approach (1000 compound in total) was assessed in vitro using the tautomerase assay. This biochemical validation process resulted in the identification of 12 novel MIF inhibitors corresponding to a $1.2 \%$ hit rate. Six of these hits came from Surflex docking; two from FlexX docking with MD simulations and four hits were identified with MDS and pharmacophore filtering with minimal overlap between the hits from each approach. Six hits were identified with $\mathrm{IC}_{50}$ values lower than $10 \mu \mathrm{M}$ (three hits with $\mathrm{IC}_{50}$ lower than $1 \mu \mathrm{M}$ ); four were shown to be suicide inhibitors and act via covalent modification of the N-terminal catalytic residues Pro1. One additional inhibitor, $N$-phenyl- $N$-1,3,4-thiadiazol-2-yl-thiourea, $\left(\mathrm{IC}_{50}=300 \mathrm{nM}\right.$ ) was obtained from FlexX docking combined to pharmacophoric filtering on one of the eight MD structures. These results demonstrate the power of integrative in silico approaches in the discovery of new modulator of MIF's tautomerase activity. The chemical diversity and mode of action of these compounds suggest that they could be used as molecular probes to elucidate the functions and biology of MIF and as lead candidates in drug developments of anti-MIF drugs.
\end{abstract}

(c) 2010 Elsevier Ltd. All rights reserved.

\section{Introduction}

Macrophage migration inhibitory factor (MIF) is among the first cytokines discovered. ${ }^{1,2}$ Initially identified as an inhibitor of the random migration of macrophages as well as a major player in delayed-type hypersensitivity, MIF was later demonstrated to play a

Abbreviations: MIF, macrophage migration inhibitory factor; wt, wild-type; SEC, size exclusion chromatography; LS, Light Scattering; MALDI, matrix-assisted laser desorption ionization; MD, molecular dynamics; HTS, high-throughput screening; F-Score, FlexX Score.

* Corresponding authors.

E-mail addresses: morelli@ifr88.cnrs-mrs.fr (X. Morelli), hilal.lashuel@epfl.ch (H.A. Lashuel).

These authors contributed equally. pivotal role in the regulation of inflammation and in host innate and adaptive immune responses. ${ }^{1-5}$ Through a range of intraand extra-cellular activities, the expression and secretion of MIF modulate immune and inflammatory responses in health and disease. ${ }^{6-9}$ MIF plays a major role in macrophage activation and phagocytosis, ${ }^{3,10-13}$ T-cell proliferation ${ }^{14}$ and antibody production by B-cells ${ }^{14}$; it also promotes cell growth, ${ }^{15,16}$ and counter-regulates glucocorticoid-induced cytokine suppression. ${ }^{17,18}$ Neutralization of MIF activity using immunological, pharmacological and/or genetic deletion approaches has shown beneficial results in a wide range of inflammatory, infectious and oncogenic disease models, ${ }^{19-24}$ thereby underlying the crucial role that MIF plays in modulating the pathogenesis of these disorders and its potential as a therapeutical target. ${ }^{6,23}$ 
Unlike other cytokines, MIF functions as an enzyme and exhibits two catalytic activities: a keto-enol tautomerase activity (substrates: D-dopachrome and (hydroxy)-phenylpyruvate) (Fig. 1A and B) and thiol oxido-reductase activity (substrates: insulin and 2-hydroxyethyldisulfide (HED)). X-ray crystallography, NMR and biophysical solution studies demonstrate that MIF exists predominantly as a homotrimer. ${ }^{25-28}$ Structure-function relationship studies demonstrated that the keto-enol isomerisation activity of MIF is catalyzed by the $\mathrm{N}$-terminal proline residue (Pro1), and involves residues from two adjacent subunits (Fig. 1C). ${ }^{29}$ Pro1 exhibits an unusual $\mathrm{p} K_{\mathrm{a}}$ of $5.6 \pm 0.1$ and is thus assumed to be un-protonated under biological conditions, which allows it to serve as a catalytic base. ${ }^{30}$ MIF shares a striking structural similarity with three bacterial isomerases: 4-oxalocrotonate tautomerase (4-OT), 5-carboxymethyl-2-hydroxymuconate isomerases (5-CHMI) and chorismate mutase. ${ }^{31}$ Like MIF, these enzymes are homotrimers (4-OT is a trimer of homodimers) and belong to the tautomerase super family characterized by a $\beta-\alpha-\beta$ motif and a catalytic amino-terminal proline. ${ }^{31}$ Mutation of the catalytic Pro1 residue in MIF, 4-OT, and 5CHMI abolishes the tautomerase activity of these enzymes. ${ }^{31,32}$

Accumulated evidence suggest that the catalytic activities of MIF play a role in modulating some of its intra- and extra-cellular activities $^{33,34}$ and its interactions with other proteins. ${ }^{35}$ Smallmolecule inhibitors of MIF tautomerase activity, such as ISO-1 ((S,R)-3-(4-hydroxyphenyl)-4,5-dihydro-5-isoxazole acetic acid methyl ester), were shown to protect against MIF proinflammatory effects in experimental models of sepsis, ${ }^{36}$ and in mouse models of endotoxemia. ${ }^{36}$ Inhibition of MIF tautomerase activity by ISO-1 inhibits the migration, invasion and anchorage-independent growth of human cancer cell lines in vitro. ${ }^{34,37,38}$ Moreover, catalytically inactive MIF mutants were unable to induce superoxide production in neutrophils, ${ }^{30}$ and displayed reduced ability to enhance the matrix metalloproteinases (MMPs) mRNA level in synovial fibroblasts from individuals with rheumatoid arthritis. ${ }^{7}$ Increased MMPs levels in serum and synovial tissue of a rheumatoid arthritis patient has been proposed as a marker of joint damage and systemic inflammation. ${ }^{39,40}$ Based on these findings, targeting the catalytic activity of MIF has emerged as an attractive and viable therapeutic strategy to attenuate its function in health and disease. The availability of extensive X-ray structural data on MIF has facilitated and accelerated the design and discovery of active site small-molecule inhibitors using structure-based approaches. The majority of the early classes of MIF inhibitors (e.g., acetaminophen, isoxazolines, and cinnamates) represent mainly analogues and derivatives of its non-physiological substrates. $^{33,36,41-43}$

Until recently, the lack of a robust high-throughput tautomerase assay, as a result of instability of its substrates, has precluded any large-scale efforts to screen large chemical libraries. Therefore, high-throughput screening (HTS) efforts have focused primarily on structure-based computer assisted approaches (in silico). ${ }^{44}$ The majority of the virtual docking methodologies applied to MIF were based on a single X-ray structure of MIF from the PDB database ${ }^{35,44}$ and the dynamics of the catalytic pocket in solution was not taken into account.

We hypothesized that a better understanding of the flexibility of the catalytic site and how it contributes to ligands binding would facilitate computer assisted drug design of small-molecules inhibitors of MIF activity and would enhance the success rates of in silico screening approaches. To test this hypothesis, we developed an original in silico protocol for the discovery of novel inhibitors targeting MIF's active site. This virtual high-throughput screening protocol takes into account the molecular dynamic parameters of MIF as well as the structural and chemical properties of its catalytic pocket. Our protocol combines three robust molecular modeling methodologies: (1) high-throughput docking of virtual compounds libraries with BioSolveIT's FlexX software version 3.0.2 applied on an ensemble of conformations generated by Molecular Dynamic (MD) simulations with CHARMM; (2) a dynamic pharmacophorebased hit selection with Tripos's Unity software; in order to eliminate irrelevant docking poses and enforce the selection of ligands interacting with key residues within the catalytic pocket of MIF and; (3) a standalone docking with the latest Surflex (version 2.4) software using the geom $X$ option that takes into account ligand flexibility during molecular docking. Our MD simulations demonstrate that the active site of MIF is quite flexible and suggested that the dynamic analysis of the protein enzymatic pocket could provide insights that would facilitate the design of novel MIF inhibitors. To validate the virtual screening protocol, we performed a biochemical experimental assay on hit candidates obtained in silico on a subset of the ChemBridge library corresponding to the best-ranked $1-3 \%$ of ligands according to their docking scores from (i) FlexX coupled to MD simulations, (ii) a combination of FlexX coupled to MD simulations and Pharmacophore Filtering

A

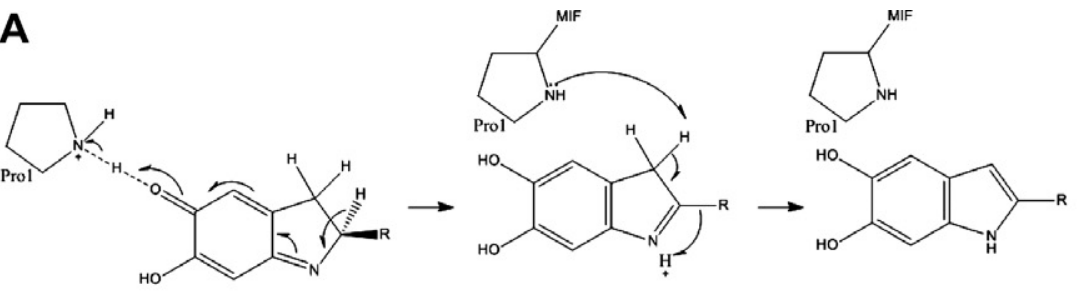

B
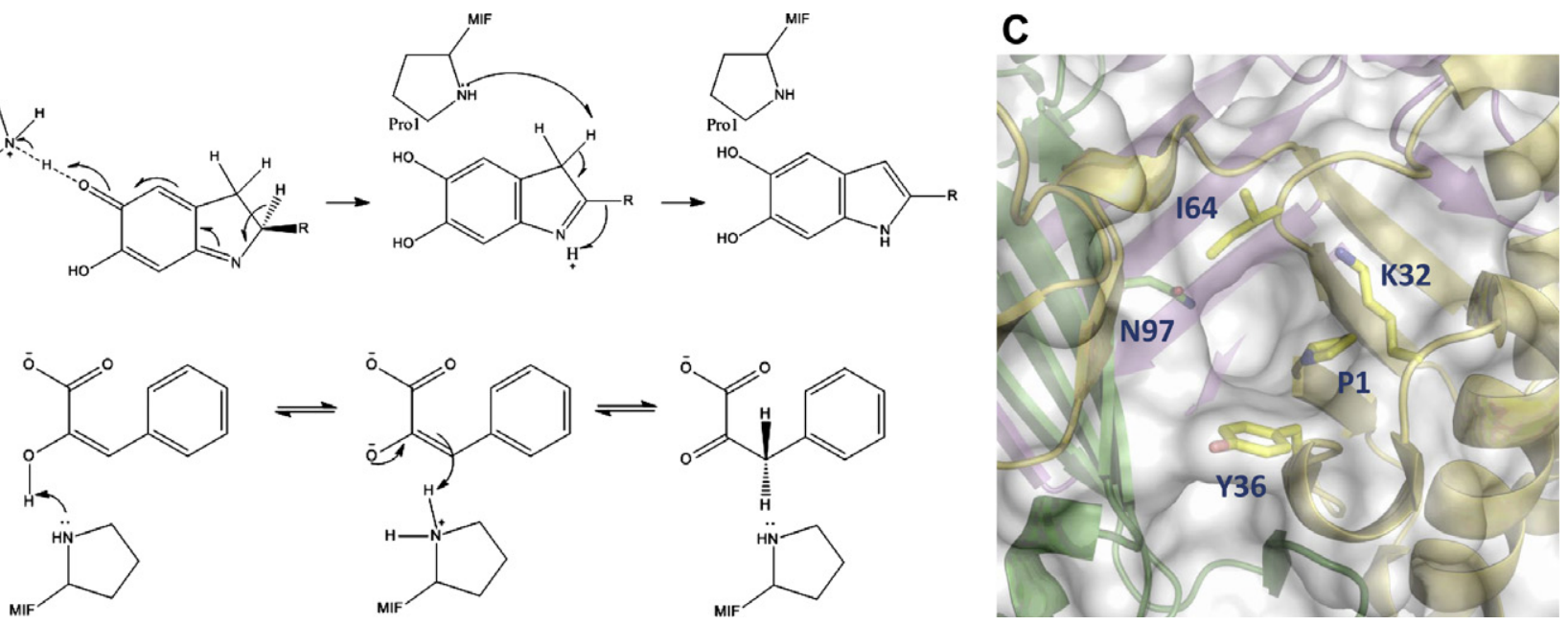

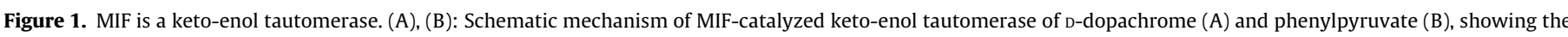

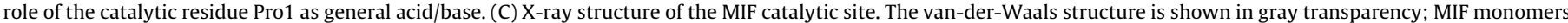

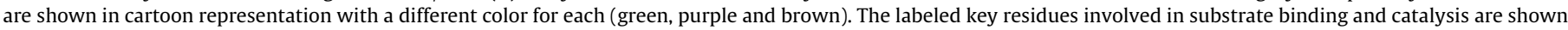
in yellow-carbon stick representation. 
(PF), or (iii) Surflex. Validation of our combined in silico protocol resulted in the discovery of three novel potent inhibitors of MIF tautomerase activity, confirmed with $\mathrm{IC}_{50} \mathrm{~S} \leqslant 1 \mu \mathrm{M}$. Nine other inhibitors were also validated with $\mathrm{IC}_{50}$ ranging from 1 to $>200 \mu \mathrm{M}$. The mechanisms of action of these inhibitors were defined using a battery of biochemical and biophysical methods. Our results suggest that coupling these different approaches developing an integrative in silico protocol should facilitate the identification and discovery of novel classes of MIF inhibitors.

\section{Results}

For well-characterized protein targets, in silico virtual screening provides rapid and low-cost complementary approaches to screen large small-molecule chemical database to identify potential inhibitors of proteins activity in vitro and in vivo. Our in silico methodology combines the power of three robust computational approaches: (1) molecular docking methods for a fast sampling of the database in the enzyme structure(s), (2) MD simulations which take into account the protein dynamics and; (3) pharmacophore filtering that allows the modeler to pilot the sampling using chosen features of the enzyme pocket. Each of these different components was first separately optimized and validated using known X-ray structures of MIF complexed with known inhibitors.

\subsection{Molecular dynamics simulations}

Structural models derived from X-ray diffraction of protein crystals yield static coordinates. Therefore, in order to account for the structural flexibility of a protein in solution, and to obtain a reasonable ensemble of MIF conformations, we performed MD simulations of MIF in explicit aqueous solvent by applying the CHARMM force field ${ }^{45-48}$ and using the 1GD0 X-ray structure as a starting template. Eight independent simulations were performed with different conditions that varied according to temperature ( 300 or $333 \mathrm{~K}$ ), presence or absence of crystallographic water molecules, and solvation (see Materials and Methods section and Supplementary Table S1). Our objective was to obtain the highest diversity possible of reasonable MIF conformations to be used as receptors in docking experiments. Coordinates were saved every 500 ps for subsequent analysis leading to a total of 32,000 conformations for the $16 \mathrm{~ns}$ simulations. The overall protein structure was stable during the time course of the trajectory, with an average root mean square deviation over CA atoms of $1.05 \pm 0.24 \AA$. In order to reduce the number of conformations to a reasonable set to be used as receptors in high-throughput docking calculations, we performed a clustering analysis of the MD ensemble. On the basis of initial visual inspection of the trajectories, we found that in a majority of the MD snapshots, the backbones and side chains of residues surrounding (Lys32, Phe113) and inside (Pro1) the active site blocked the entrance of the active site, thus preventing any docking. Therefore, the clustering analysis was aimed at separating the 'open' from 'closed' conformations. The criteria used were a set of distances described in Supplementary Table S2. We obtained 78 structures fulfilling these criteria, which we visually inspected in detail. From these, we selected 55 diverse MIF structures that were used to optimize the virtual HTS protocol (Table 1).

\subsection{Optimization of the virtual screening protocol}

Whereas MD simulations were used to model the protein's flexibility in solution, docking was used to account for the possibility that bound ligands may adopt conformations distinct from their minimum free energy states. Docking also served the purpose of ranking the ligands according to a scoring function. Several scoring
Table 1

Comparison of the available crystal structures of MIF in the PDB, and the eight conformations obtained by MD simulations and used for pharmacophoric filtering

\begin{tabular}{lll}
\hline Structures compared & RMSD mean $(\AA)$ & RMSD Stdev $(\AA)$ \\
\hline 1GD0/X-ray & 0.186 & 0.053 \\
1GD0/MDS & 0.759 & 0.126 \\
\hline
\end{tabular}

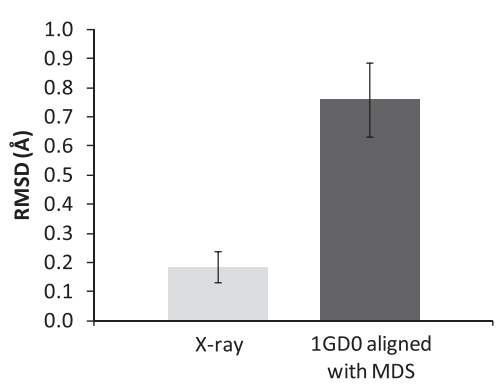

The ligand-free X-ray structure $1 \mathrm{GD0}$ is very similar to structures in which ligands were co-crystallized (1CA7, 1GCZ, 1LJT, 1MFI, 2OOZ), but the RMSD between $1 \mathrm{GD} 0$ and the MD structures is significantly higher $\left(p=2.1 \times 10^{-7}\right)$.

functions were available and tested: F-Score (FlexX's scoring function), D-Score, G-Score, PMF-Score, and Chem-Score. The first step in optimizing the virtual screening process was thus to select the scoring function that best correlated the calculated score with the crystallographic structures available. To this end, we selected entries from the PDB in which MIF was co-crystallized with a known substrate (4-hydroxyphenylpyruvate, PDB $1 C A 7^{29}$ ) or inhibitors (7-hydroxy-2-oxo-chromene-3-carboxylic acid ethyl ester, PDB $1 G C Z, 44$ 3-(4-hydroxyphenyl)-4,5-dihydro-5-isoxazole-acetic acid methyl ester, PDB 1LJT, ${ }^{41}$ 2-fluoro-3-(4-hydroxyphenyl)-2E-propeneoate, PDB $1 \mathrm{MFI},{ }^{42}$ and 4-hydroxybenzaldehyde $o$-(3,3-dimethylbutanoyl)-oxime, PDG $200 Z^{49}$ ). We then re-docked each ligand into its corresponding pocket, consisting of the same ensemble of residues as specified in Materials and Methods (Table 2). The best poses were those having an RMSD of less than $2.0 \AA$ with respect to the X-ray coordinates. For each receptor-compound complex, we scored the best 30 poses according to each function mentioned above, and chose as the best scoring function the one that gave the best scores to the poses with the lowest RMSDs. We found that FScore gave higher scores to the poses that were most similar to the crystal structure (data not shown).

Although scoring is a critical part of molecular docking, its main purpose in our protocol was to generate reasonable conformations of screened small-molecules inside the catalytic pocket of MIF. From the database of poses computed by FlexX, candidate inhibitors were selected through a pharmacophore-based filtering step. A receptor-defined model of MIF's tautomerase site was built with the Unity software. Briefly, it consists of a 'negative image' of the catalytic pocket's features, such as hydrogen bond donors and acceptors, in the form of 3D spatial constraints into which screened compounds must fit to be selected as hits. The residues used to define the pharmacophore are detailed in Materials and Methods and shown in Figure 2. We then optimized the tolerance of these spatial features, that is, the allowed distance from an ideal pose. The best tolerance is a compromise between the ability of the pharmacophore to reject false positives and its capacity to retain as many true positives as possible. To determine this value, we built a series of pharmacophores with tolerances ranging from 0.5 to 2.0 Å for each of the 55 MIF conformers obtained by MD simulations. The 55 conformers were first docked with the 5 positive control ligands (mentioned above) and 200 true negatives to generate $30 \times 205=6150$ poses. Each generated model (6150 poses) was then filtered through the 55 pharmacophores corresponding to tolerances from 
Table 2

Structures and binding modes of positive control ligands that were co-crystallized with MIF

\begin{tabular}{|c|c|c|}
\hline Ligand & Structure & Residue:atom \\
\hline p-Hydroxyphenylpyruvate (1CA7) & & N97:OD1, N97:HD21, N97:HD22, K32:HZ1-3, I64:HN \\
\hline Chromene (1GCZ) & & N97:OD1, N97:HD21, N97:HD22, K32:HZ1-3, I64:HN \\
\hline ISO-1 (1LJT) & & N97:OD1, N97:HD21, N97:HD22, K32:HZ1-3 \\
\hline 2-FHC (1MFI) & & N97:OD1, N97:HD21, N97:HD22, K32:HZ1-3, P1:N \\
\hline OXIM-6 (200Z) & & N97:OD1, N97:HD21, N97:HD22, Y95:OH, Y36:O \\
\hline
\end{tabular}

For each ligand, the key protein residues interacting with these compounds are shown, along with the respective atoms involved.

A

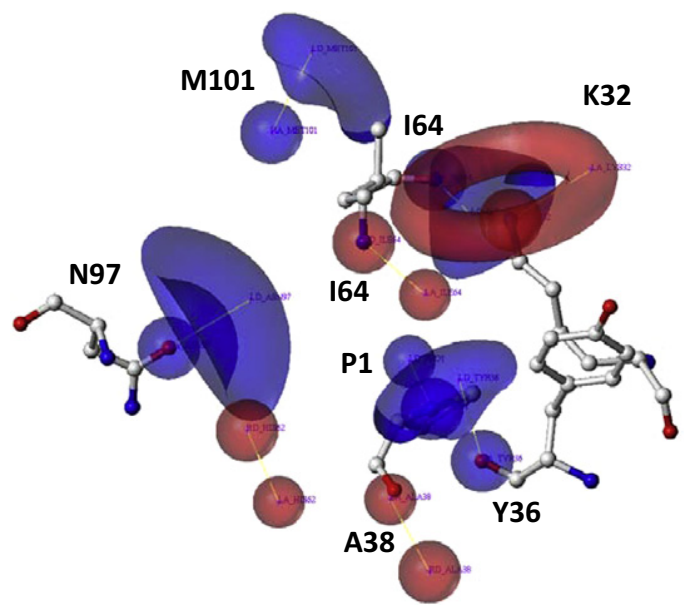

C

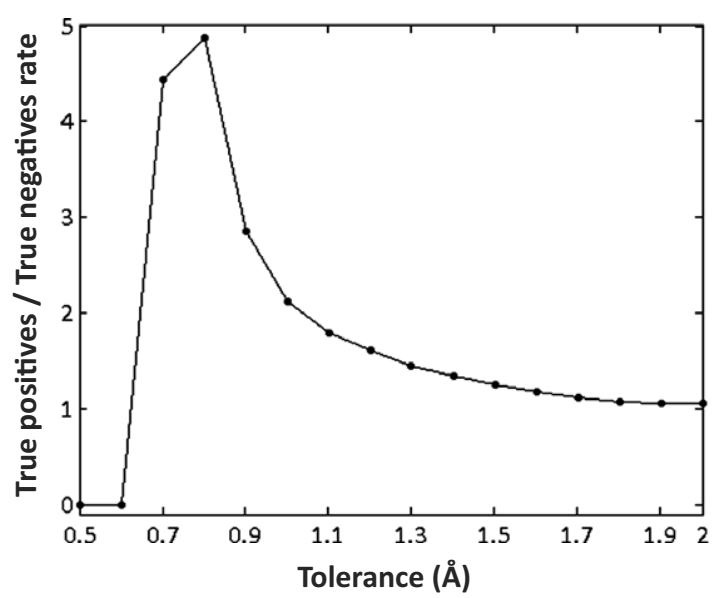

B

Proline 1, N

Tyrosine36, 0

Protein Acceptors' sites Isoleucine 64, 0

Asparagine 97, OD1

Methionine 101, o

Lysine 32, NZ

Alanine $38, \mathrm{NH}$

Histidine 62, NH

Isoleucine $64, \mathrm{NH}$

D

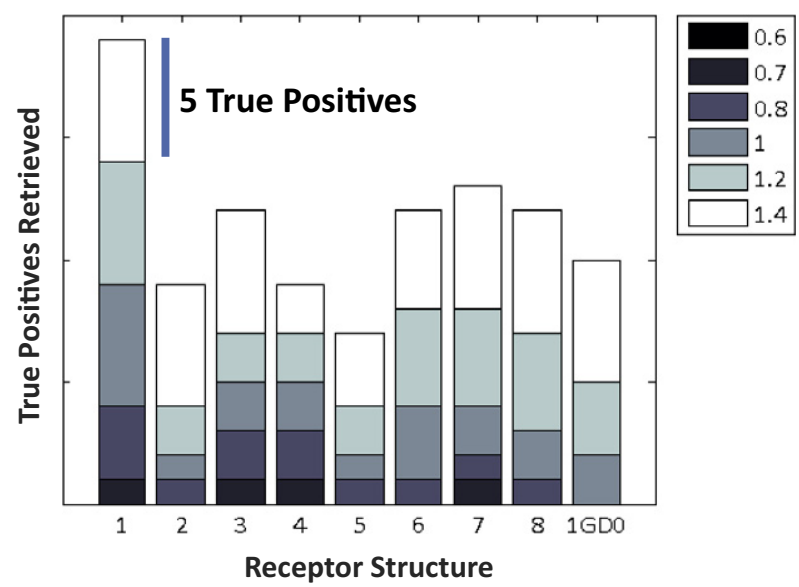

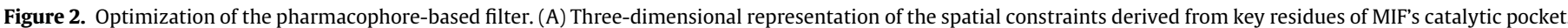

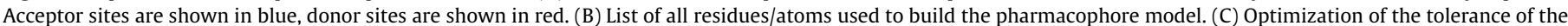

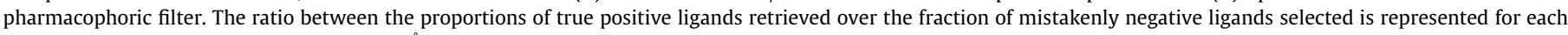

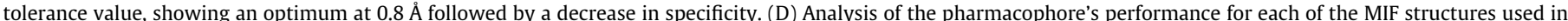

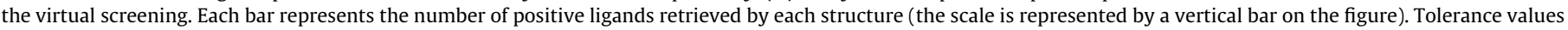
from 0.6 to $1.4 \AA$ are mapped to the bar colors. Note that at tolerance $0.8 \AA$ all five of the true positives are retrieved with the eight MD structures. 
0.5 to $2 \AA$. The lowest tolerance for which all five controls were retrieved with the lowest number of true negatives was selected. Figure $2 \mathrm{C}$ shows the number of retrieved true positive hits as a function of pharmacophore tolerance. With this procedure, we obtained an optimal tolerance of $0.8 \AA$. This step allowed us to further reduce the number of MIF conformers to be used in the final virtual screening. At the optimal tolerance of $0.8 \AA$, we found that eight MD structures (with their corresponding pharmacophore) were sufficient to retrieve the set of five structurally diverse control ligands (Fig. 2D). These eight structures (Fig. 3) were chosen as templates for our screening protocol, in addition to the X-ray structure. The remaining structures (47) required higher tolerances and were discarded.

\subsection{Virtual high-throughput screening}

With the optimized in silico pipeline (F-score as scoring function, 1GD0 + eight MD protein conformers and tolerance ratio of $0.8 \AA$ ), we proceeded to screen for novel candidate MIF inhibitors from the Chembridge library, comprising $\sim 500,000$ compounds. We reduced this number to 33,900 by applying a restriction of 30 or fewer non-hydrogen atoms, based on the observation that the binding pocket can only accommodate low molecular weight compounds and that known MIF inhibitors always fulfill this condition. ${ }^{35}$ Docking experiments also showed that larger molecules are generally bound outside the active site (data not shown).

The 33,900 molecules database was docked against each of the eight 'optimal' MIF conformers obtained by MD simulations, plus the 1GD0 structure (Fig. 4). The 30 best poses for each compound were selected according to the FlexX scoring function (F-Score) resulting in $\sim 9,000,000$ structures $(33,900 \times 9$ structures $\times 30$ poses). Each generated model was then subjected to our pharmacophore-based filtration using the optimized tolerance value of 0.8 A on the nine templates (1GD0 + eight MD structures). We considered as potential hits the structures matching at least two protein acceptor sites and one protein H-bond donor site, with the condition that one of these matched features must be at the interior of the catalytic site (Fig. 2A and B). At this stage, our objective was to reject poses that were docked onto the rim of the tautomerase pocket of the protein. After eliminating duplicates (i.e., conformers of the same compound), we obtained 5185 unique potential hits.

Previous studies by our group have demonstrated robust results using Surflex as docking software. After a validation process that consisted of checking that Surflex could retrieve all the correct poses (RMSD $<2 \AA$ ) from the single docking with the different Xray structures (1CA7, 1GCZ, 1LJT, $1 \mathrm{MFI}$ and 200Z), Surflex highthroughput docking was carried out on MIF apo-structure (PDB code 1GD0) using the same amino acids as described for the FlexX docking to generate the 'Surflex protomol' (Surflex priority area of docking) with standard parameters (threshold 0.5 , no bloat). It is noteworthy that at this stage only a very small overlap was observed between the 333 hits first selected by Surflex and those selected either from FlexX on the nine representative structures or those selected from FlexX on the nine representative structures filtered by the pharmacophore approach. This validates the necessity of coupling the best $1 \%$ from each approach into a global in silico selection that should increase the chemical diversity of our resulting hits.

\subsection{Experimental HTS evaluation of the best 1000 compounds obtained using our integrative in silico protocol}

To validate our in silico protocol(s) and to test the inhibitory effects of the highly-ranked molecules identified, we chose the bestscored 1000 molecules to be experimentally validated via the HTS protocol employing the tautomerase assay recently developed in our laboratory. ${ }^{50}$ The 1000 -compounds set consisted of the following: (i) best 333 scored-molecules selected using the 'Docking standalone' (Surflex) protocol; (ii) best 333 scored-molecules selected using the 'Docking + MDS' (FlexX score + CHARMM), but not retrieved by Unity filtering; and (iii) best 334 scored-molecules selected using the 'Docking + MDS + Unity Pharmacophore Filtering' (FlexX score + CHARMM + Unity). D-dopachrome substrate was selected for the screening. As a positive control, we used benzyl isothiocyanate (BITC), which we recently reported as a potent inhibitor of MIF that leads to complete inhibition of MIF tautomerase activity at $10 \mu \mathrm{M} .{ }^{52}$ Robustness and reproducibility of the assay were determined through calculations of the intra- and inter-plate
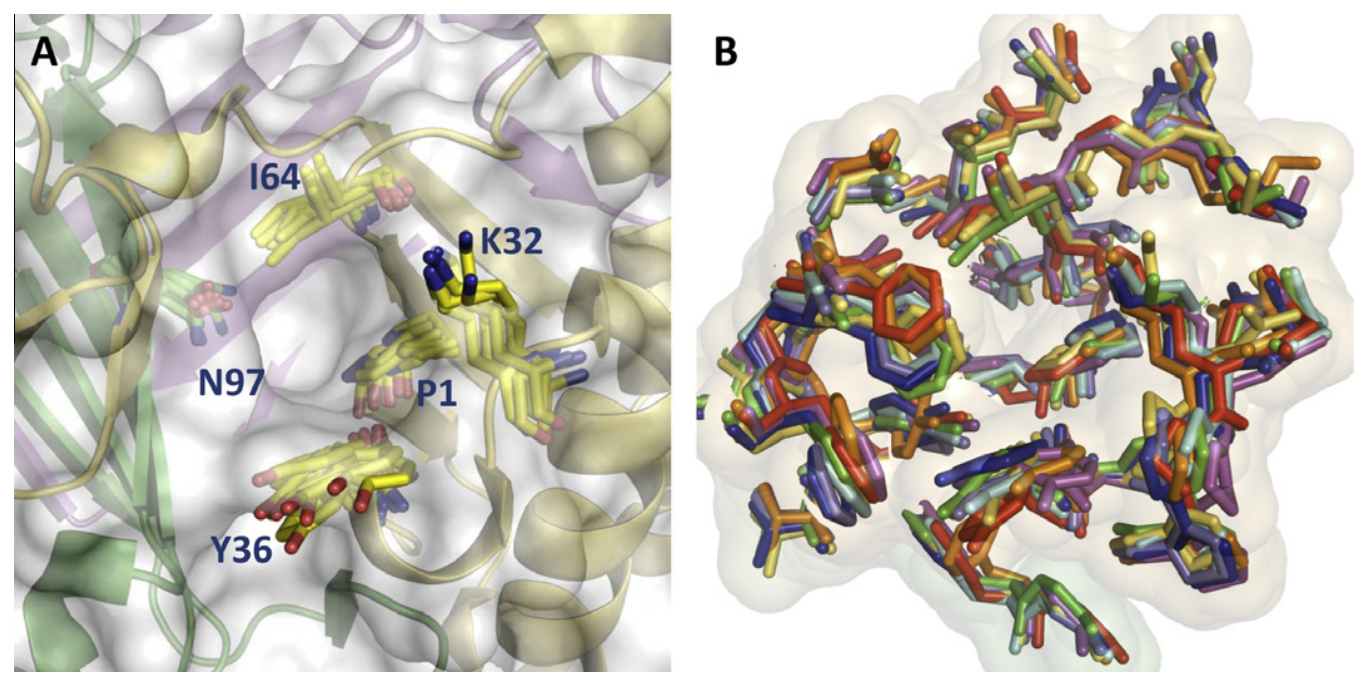

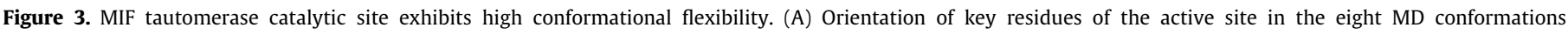

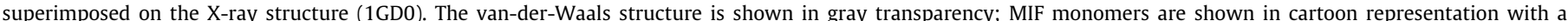

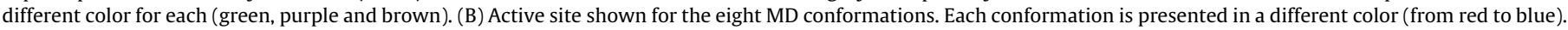
Chains $B$ and $C$ surfaces are represented in green and pale orange, respectively. 


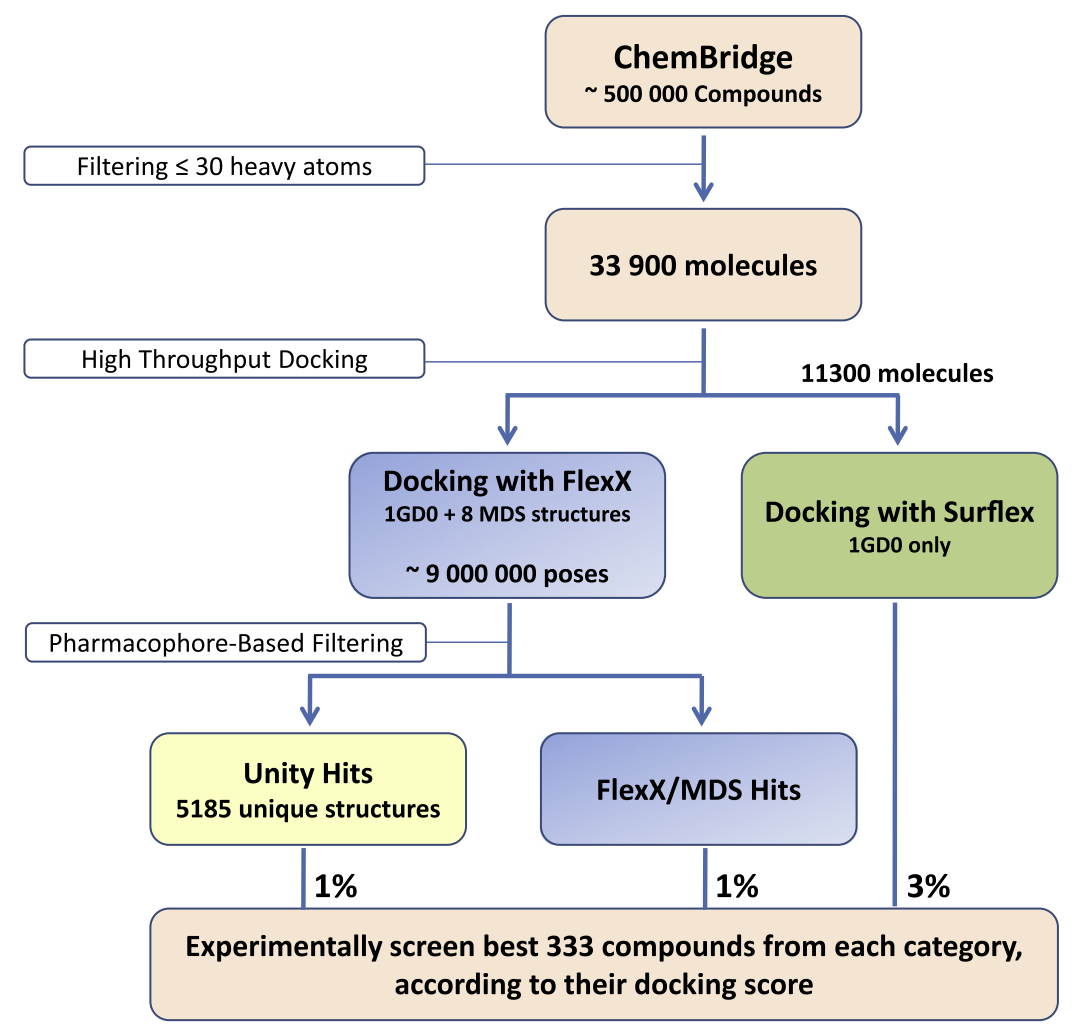

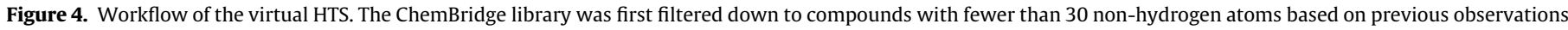

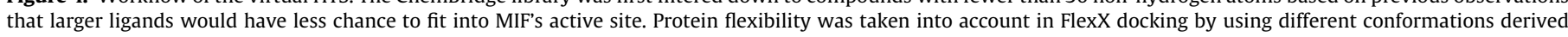

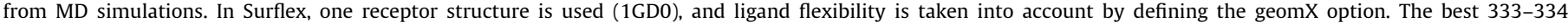
compounds from each category, corresponding to a total of 1000 compounds were validated by experimental HTS.

variations using single-point $Z^{\prime}$ measurements, which yielded a $Z^{\prime}$ of $\geqslant 0.9$ for the assay.

The 1000 compounds were screened at two different concentrations in 384 multi-well plates; the assay was performed as recently described in Ouertatani-Sakouhi et al. ${ }^{50}$ Briefly, the protein was incubated with 10 or $100 \mu \mathrm{M}$ of compound for an hour at RT (final DMSO percentage $\leqslant 1.5 \%$ ). The reaction was then initiated by the addition of D-dopachrome. It is noteworthy that DMSO at $\leqslant 2 \%$ has no effect on MIF tautomerase activity. ${ }^{50}$ In the absence of MIF, the absorbance of the substrate at $475 \mathrm{~nm}$ is around $0.9-1$. Upon addition of MIF, the absorbance decreases to $\sim 0.1$ within $3 \mathrm{~min}$. Inhibitor potencies were measured by recording the absorbance at the end point ( $2 \mathrm{~min}$ ). Hit compounds showing greater than $15 \%$ inhibition at $100 \mu \mathrm{M}$ were selected and validated manually to eliminate the false positives (Table 3 ). A further validation was performed, in order to determine whether some of the confirmed inhibitors could act via an aggregation-based, non-specific mechanism. The assay was adapted from Feng and Shoichet. ${ }^{51}$ Briefly, it consists of comparing the $\mathrm{IC}_{50}$ s measured in absence and presence of low amounts of non-ionic detergent. Compounds that show a strong reduction in inhibition upon addition of detergent are likely to be aggregators and are not counted as computational successes, as they are not true binders. We chose to use $0.1 \%$ $\mathrm{v} / \mathrm{v}$ Triton X-100 as detergent in both assay buffer and enzyme stock solution, after we showed this concentration of Triton has no effect on MIF's tautomerase activity (data not shown). The results are shown in Figure 5. Addition of detergent fully reversed inhibition in the case of hits 5,9 , and 14 , indicating a probable mechanism based on a phase transition of these inhibitors. Among the remaining compounds, three were confirmed with $\mathrm{IC}_{50} \mathrm{~S} \leqslant 1 \mu \mathrm{M}$ (hit 1: $50 \mathrm{nM}$, hit 2: $300 \mathrm{nM}$, and hit 3: $80 \mathrm{nM}$ )
(Fig. 5, Table 3) and 9 others with $\mathrm{IC}_{50} \mathrm{~S}$ ranging from 1 to $>200 \mu \mathrm{M}$ (Table 3).

\subsection{Mode of action of MIF antagonists}

To assess the mode of action of the validated hits (i.e., excluding hits 5, 9, and 14) obtained in our screen, we performed a series of biochemical and biophysical studies to determine whether the inhibitors exert their effects by one of the following mechanism: (1) covalent modification of the N-terminal catalytic Pro1 residue; (2) direct binding to the active site; (3) alteration of the tertiary structure of MIF, or; (4) disruption of the trimer. Each hit compound was incubated with MIF (1/1 molar ratio) at rt for one hour and the reaction mixtures were evaluated by MALDI/TOF-TOF mass spectrometry, size exclusion chromatography, light scattering and SDS-PAGE analysis. None of the compounds tested caused disruption of the trimer or affected the solubility of MIF (data not shown). To determine whether the selected compounds covalently modify MIF, the reaction mixtures were analyzed by MALDI/TOFTOF tandem mass spectrometry. Potent hits obtained by Surflex standalone docking, hits $1\left(\mathrm{IC}_{50}=50 \mathrm{nM}\right)$ and $3\left(\mathrm{IC}_{50}=80 \mathrm{nM}\right)$, as well as hits $4\left(\mathrm{IC}_{50}=2.8 \mu \mathrm{M}\right)$ and $6\left(\mathrm{IC}_{50}=6.9 \mu \mathrm{M}\right)$ obtained by docking with FlexX on MD structures, resulted in a shift in the molecular mass of MIF (Fig. 7) that was consistent with a single modification of MIF by these compounds. To identify the exact residue modified by these inhibitors, the modified MIF species were subjected to tryptic digestion and peptide mapping by MALDI/ TOF-TOF mass spectrometry. In every case, we observed a molecular weight shift corresponding to a modified N-terminal fragment comprising residues PMFIVNTNVPR (MW = 1287.6 Da) (Fig. 7). MS/ MS analyses and sequencing of the modified peptide fragment 
Table 3

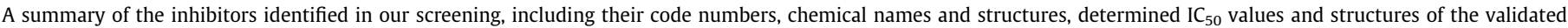
inhibitors

\begin{tabular}{|c|c|c|c|c|c|c|}
\hline $\begin{array}{l}\text { Hit } \\
\text { names }\end{array}$ & Origin & $\begin{array}{l}\text { Catalog } \\
\text { ID }\end{array}$ & Compound name & $\begin{array}{l}\mathrm{MW}(\mathrm{g} / \\
\mathrm{mol})\end{array}$ & $\begin{array}{l}\mathrm{IC}_{50}(\mu \mathrm{M}) \text { or max. } \\
\text { inhibition }(\%)\end{array}$ & Structure \\
\hline 1 & Surflex & 7956004 & 6-Phenyl-3-pyridazinyl 2-thiophenecarboxylate & 282.3 & $0.05 \mu \mathrm{M}$ & \\
\hline 2 & Unity & 5484950 & $N$-Phenyl- $N^{\prime}$-1,3,4-thiadiazol-2-ylthiourea & 236.3 & $0.3 \mu \mathrm{M}$ & \\
\hline 3 & Surflex & 5320163 & S-1,3-Benzoxazol-2-yl O-butyl thiocarbonate & 251.3 & $0.08 \mu \mathrm{M}$ & \\
\hline 4 & FlexX & 6205546 & $N$-[(2-Bromobenzoyl)oxy]-4-chlorobenzamide & 354.6 & $2.8 \mu \mathrm{M}$ & \\
\hline 5 & Surflex & 6367607 & $\begin{array}{l}\text { 5-(\{2-Nitro-4-[(trifluoromethyl)sulfonyl }] \text { phenyl }\} \text { thio })-1,3,4- \\
\text { thiadiazole-2(3H)-thione }\end{array}$ & 403.4 & Detergent-sensitive & \\
\hline 6 & FlexX & 7291474 & 1-(2-Methoxybenzoyl)-1H-1,2,3-benzotriazole & 253.3 & $6.9 \mu \mathrm{M}$ & \\
\hline 7 & Surflex & 9049826 & $\begin{array}{l}\text { Methyl \{[4-methyl-5-(2-thienyl)-4H-1,2,4-triazol-3- } \\
\text { yl]thio\}acetate }\end{array}$ & 269.3 & $18.6 \mu \mathrm{M}$ & \\
\hline 8 & Surflex & 5113769 & 3-Acetyl-7-hydroxy-2H-chromen-2-one & 204.2 & $45.2 \mu \mathrm{M}$ & \\
\hline 9 & FlexX & 6598745 & $N$-(2-Furylmethyl)-7-nitro-2,1,3-benzoxadiazol-4-amine & 260.2 & Detergent-sensitive & \\
\hline 10 & Surflex & 5315866 & Ethyl 2-cyano-3-(2,4-dihydroxyphenyl)acrylate & 233.2 & $62.1 \mu \mathrm{M}$ & \\
\hline 11 & Unity & 5185917 & $N$-(1H-1,2,3-Benzotriazol-1-ylmethyl)-2-pyridinamine & 225.3 & $69 \%(200 \mu \mathrm{M})$ & \\
\hline 12 & Unity & 7847308 & $N$-(2-Chlorophenyl)- $N^{\prime}$-2-pyridinylthiourea & 263.8 & $1.04 \mu \mathrm{M}$ & \\
\hline 13 & Surflex & 7509861 & $\begin{array}{l}\text { 3-[(2,4-Difluorophenyl)amino]-1-(5-methyl-2-furyl)-2-propen- } \\
\text { 1-one }\end{array}$ & 263.2 & $57.6 \mu \mathrm{M}$ & \\
\hline 14 & FlexX & 5140738 & 3-Nitroso-2-phenylindolizine & 222.2 & Detergent-sensitive & \\
\hline 15 & Unity & 6150973 & 3-Benzoyl-7-hydroxy-2H-chromen-2-one & 266.3 & $64.2 \mu \mathrm{M}$ & \\
\hline
\end{tabular}

revealed that modification by these compounds takes place exclusively at the $\mathrm{N}$-terminal proline residue.

\subsection{Covalent modification of MIF by hits $1,3,4$ and 6 alter the tertiary structure and SEC elution profile of the trimer}

Previous studies from our laboratory demonstrated that MIF exists as a mixture of two different trimeric states that differ at the level of tertiary structure; this mixture elutes from the SEC column as two peaks, each of which corresponds to the MIF trimer. ${ }^{28}$ More recently we showed, using NMR, SEC, and light scattering, that covalent modification of MIF by isothiocyanates (ITCs) alters the tertiary structure of the trimer and results in a shift in the elution profile of MIF, causing it to elute earlier as a single peak. ${ }^{52}$ To determine the effect of the selected hit compounds on MIF tertiary and quaternary structures, the reaction mixtures (MIF + hits) were 


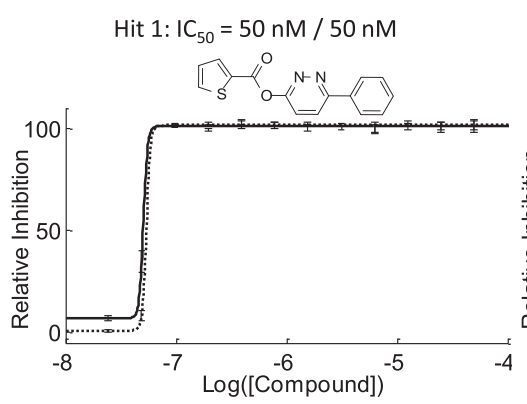

Hit 4: $I C_{50}=2.8 \mu \mathrm{M} / 4.2 \mu \mathrm{M}$

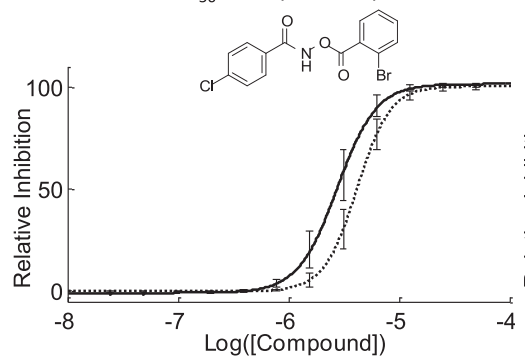

Hit 7: $I_{50}=18.6 \mu \mathrm{M} / 28.4 \mu \mathrm{M}$

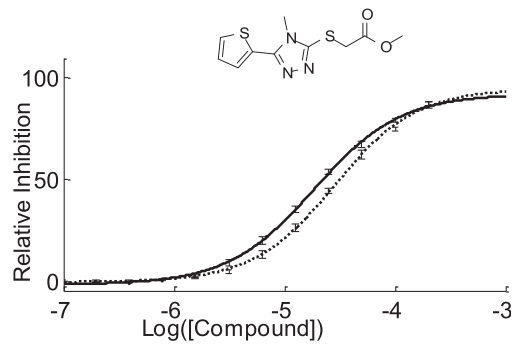

Hit 10: $I C_{50}=62.1 \mu \mathrm{M} / 110.2 \mu \mathrm{M}$

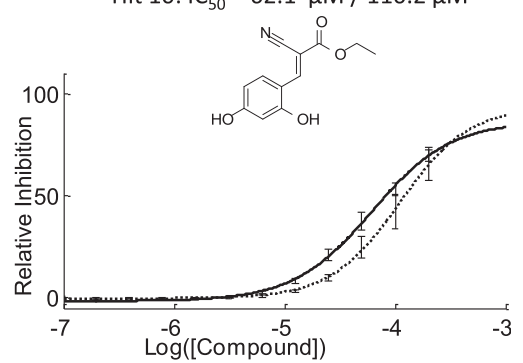

Hit 13: $I C_{50}=57.5 \mu \mathrm{M} /$ N.D.

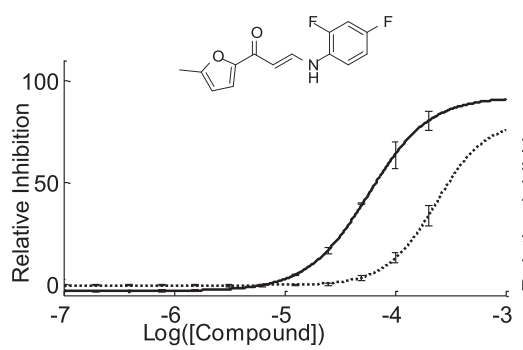

Hit 2: $I C_{50}=300 \mathrm{nM} / 420 \mathrm{nM}$

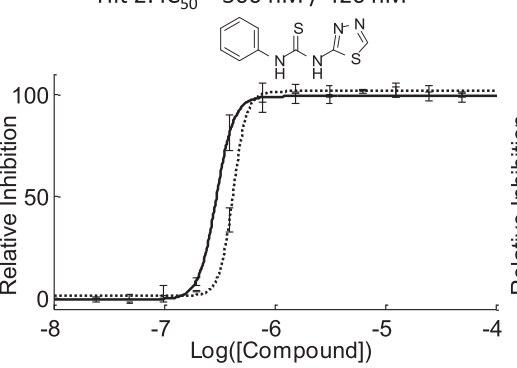

Hit 5: $I_{50}=9.5 \mu \mathrm{M} / \mathrm{N} . \mathrm{D}$.

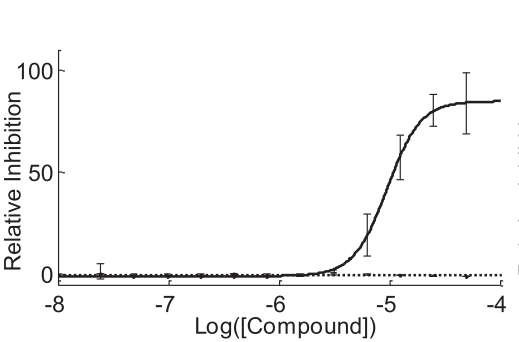

Hit 8: IC $\mathrm{C}_{50}=45.2 \mu \mathrm{M} / 103.2 \mu \mathrm{M}$
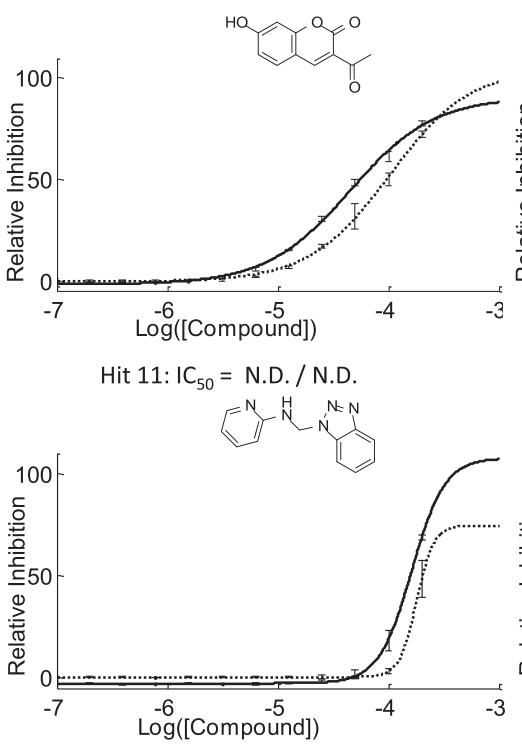

Hit 14: IC $50=120.8 \mu \mathrm{M} /$ N.D.

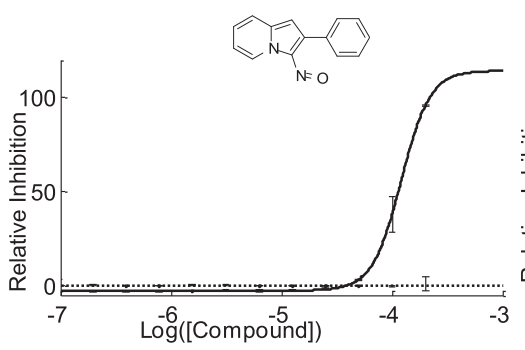

Hit 3: $I C_{50}=80 \mathrm{nM} / 150 \mathrm{nM}$

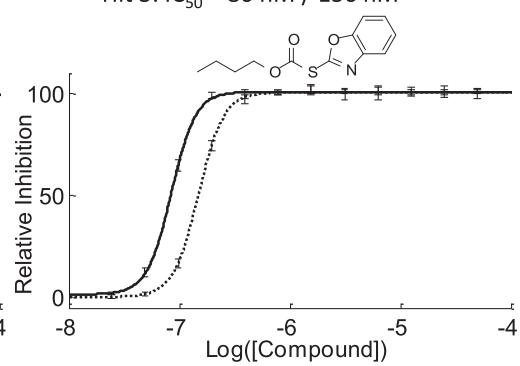

Hit $6 \mathrm{IC}_{50}=6.9 \mu \mathrm{M} / 12.2 \mu \mathrm{M}$

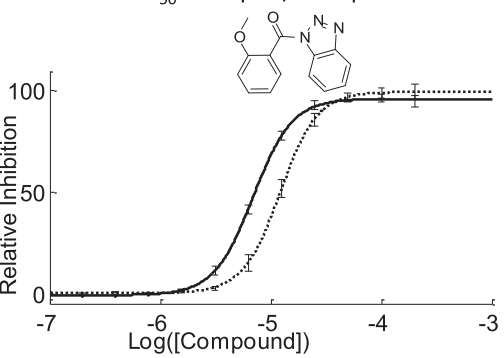

Hit 9: $I C_{50}=60.4 \mu \mathrm{M} / \mathrm{N} . \mathrm{D}$.

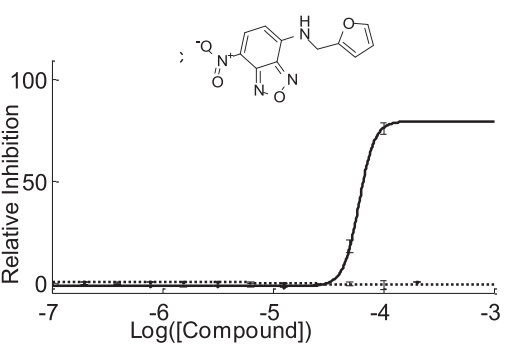

Hit 12: $\mathrm{IC}_{50}=1.04 \mu \mathrm{M} / 1.01 \mu \mathrm{M}$

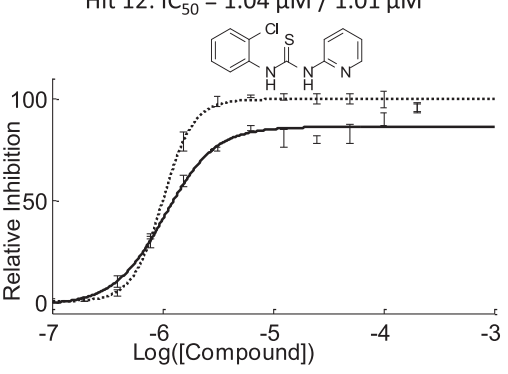

Hit 15: $I_{50}=64.2 \mu \mathrm{M} / \mathrm{N} . \mathrm{D}$.

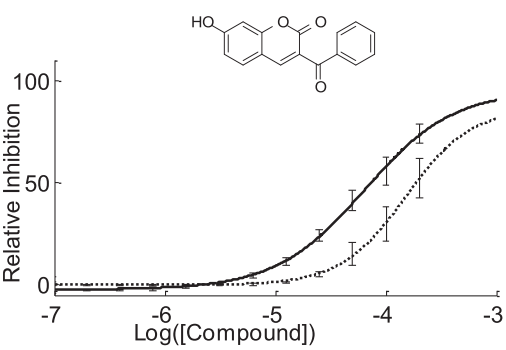

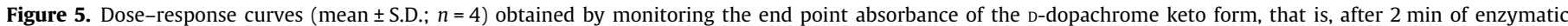

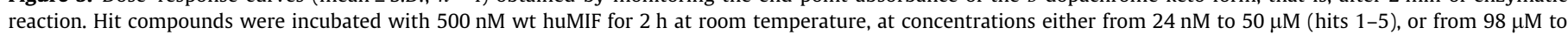

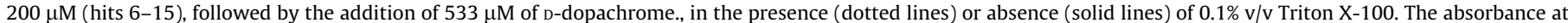

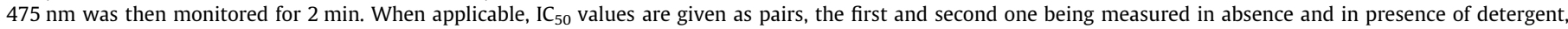
respectively. N.D., not determined.

examined by analytical size exclusion chromatography coupled to a multiangle light scattering detector. We observed that inhibition and covalent modification of MIF with hits 1, 3, 4 and 6 led to a major shift in the MIF elution profile, causing it to elute as a single peak with an earlier elution time (between 28 and 30 min) than that of unmodified MIF, which eluted as two overlapping peaks with elution times of 30.5 and $32.5 \mathrm{~min}$. These changes in the elution profile of MIF are similar to those we observed after 

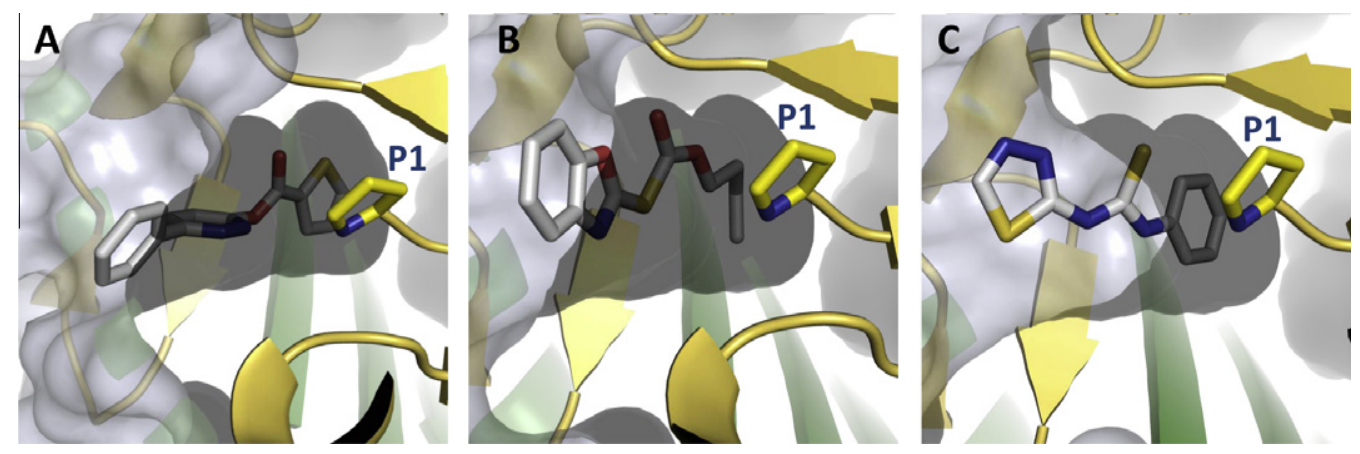

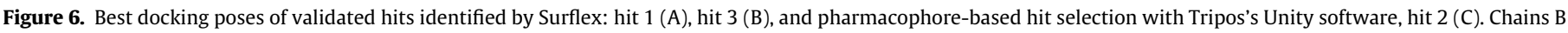
and $\mathrm{C}$ are represented in green and gold, respectively. The compounds are represented in the best score most populated conformational state.

incubation of the protein with benzyl isothiocyanate (BITC) and other isothiocyanate-based inhibitors of $\mathrm{MIF}^{52}$ (Fig. 8). It is noteworthy, that the degree of peak shift correlated well with the potency of the inhibitor and with the extent of MIF modification by each inhibitor as determined by mass spectrometry. MIF incubated with hits $1\left(\mathrm{IC}_{50}=50 \mathrm{nM}\right)$ and hit $3\left(\mathrm{IC}_{50}=80 \mathrm{nM}\right)$ eluted at $\sim 28$ min, whereas MIF incubated with hit $6\left(\mathrm{IC}_{50}=11.86 \mu \mathrm{M}\right)$ eluted $\sim 29.5 \mathrm{~min}$, and MIF incubated with hit $4\left(\mathrm{IC}_{50}=2.8 \mu \mathrm{M}\right)$ eluted at $\sim 30$ min. Other hits (e.g., hit 3) did not affect MIF's elution profile, despite their potency, suggesting that their binding does not affect MIF's tertiary and conformational structure.

In summary, we have validated a total of 12 hits, out of 1000 compounds tested, and corresponding to a $1.2 \%$ hit rate. Six of these hits come from Surflex docking, two from FlexX docking with MDS and four hits were identified with MDS and Unity (Tables 3 and 4). In another experimental HTS of the Maybridge database using the same experimental protocol, we validated 15 hits out of 14,400 compounds tested, corresponding to a $0.104 \%$ hit rate. We also screened the FDA library and have validated 2 hits out of 1400 compounds, corresponding to a $0.19 \%$ hit rate. It should be noted here that the FDA library consists of compounds that received FDA approval; these are smaller than those found in the Maybridge library and thus it is not surprising that we validated more hits in a blind test from this library. We should thus expect a $0.104 \%$ hit rate from a blind screening of the Chembridge library. It follows that when comparing our integrative in silico approach versus with blind experimental screening the global enrichment factor is around 12 (Table 4). This result demonstrates the high productivity of in silico procedures in such biological systems.

\subsection{Specificity of the suicide inhibitors}

To determine whether covalent modification of MIF's Pro1 residue by hits $1,3,4$, and 6 was specific to MIF, and possibly requires a specific binding to the active site, we incubated $10 \mu \mathrm{M}$ of each inhibitor with (1) four structurally unrelated proteins (at $10 \mu \mathrm{M}$ ) that contain lysine and/or cysteine residues: RNAse A, lysozyme, chymotrypsinogen A and ubiquitin (Supplementary Fig. S4C-F); and (2) the bacterial MIF homolog 4-oxalocrotonate tautomerase (4-OT), (Supplementary Fig. S4B). The compound-protein mixtures were incubated at room temperature for $1 \mathrm{~h}$ in MIF D-dopachrome assay buffer. None of the four inhibitors (hits 1, 3, 4, and 6) was observed to modify RNAse A, lysozyme, chymotrypsinogen A and ubiquitin as determined by mass spectrometry (Supplementary Fig. S4). These findings support the specificity of these molecules for MIF and suggest that covalent modification of Pro1 by these compounds is likely to be mediated by their binding to the catalytic site and/or the high nucleophilicity of Pro1. In the case of 4-OT, which shares a catalytic N-terminal proline residue with similar properties as that of $\mathrm{MIF}^{53}$ we observed mass shifts of 109 and $100 \mathrm{Da}$ upon incubation with the hits 1 and 3, respectively. These findings are expected and consistent with the highly nucleophilic properties of Pro1 in MIF and 4-OT.

\section{Discussion}

MIF has emerged as a major therapeutic target in a wide range of inflammatory, autoimmune and oncogenic diseases. Current therapeutic strategies for neutralizing MIF activity are focused on immunoneutralization using anti-MIF antibodies or on pharmacological approaches aimed at targeting the catalytic site. Although both strategies have shown beneficial effects in different experimental models of inflammatory, infectious and autoimmune diseases, ${ }^{34,54-56}$ the two strategies target different biochemical and cellular activities of MIF. Anti-MIF antibodies are effective in neutralizing MIF activity by blocking its interaction with other proteins and receptors. However, the tautomerase activity of MIF is not significantly affected upon formation of the MIF-antibody complex (Ouertatani-Sakouhi $\mathrm{H}$ and Lashuel $\mathrm{H}$, unpublished results). By contrast, several studies have shown that small-molecule inhibitors of MIF tautomerase activity, while targeting the active site, can induce significant tertiary structure changes in the trimer such that antibody recognition of MIF and its receptor binding properties are altered. ${ }^{35}$ These findings suggest that different active site inhibitors may have different effects on the intra- and extra-cellular activities of MIF, depending on how they modify the tertiary and/or quaternary structure of the protein.

The majority of MIF active site inhibitors developed to date were identified by screening substrate analogues or derivatives of known MIF substrates and inhibitors. ${ }^{33,36,41-43}$ The lack of a robust high-throughput assay, as a result of the low stability of known MIF substrates, has precluded the screening of large chemical libraries. Computational and molecular docking approaches provide unique opportunities for high-throughput screening and exploration of large chemical landscapes, including chemical spaces that are not yet accessible by synthetic chemistry, with minimal time and resources. To identify diverse classes of inhibitors, several groups employed computational approaches and docking of chemical libraries to the tautomerase active site. In 2002 , Orita et al. performed a virtual screening of one million compounds via molecular docking with the роск4.0.1 docking program. Five hundred twenty-four compounds were then selected, purchased and tested in vitro ${ }^{57}$; fourteen inhibitors with $K_{\mathrm{i}}$ values ranging between 0.038 and $7.4 \mu \mathrm{M}$ were validated. Recently, Winner and colleagues identified 4-iodo-6-phenylpyrimidine, 4-IPP ( IC $\left._{50}=5 \mu \mathrm{M}\right)$, as a suicide inhibitor of MIF tautomerase activity, via virtual screening using Ludi (Accelrys software). ${ }^{34}$ To discover 
$\mathrm{N}$ terminus sequence: PMFIVNTNVPR, MW = 1287.6 Da
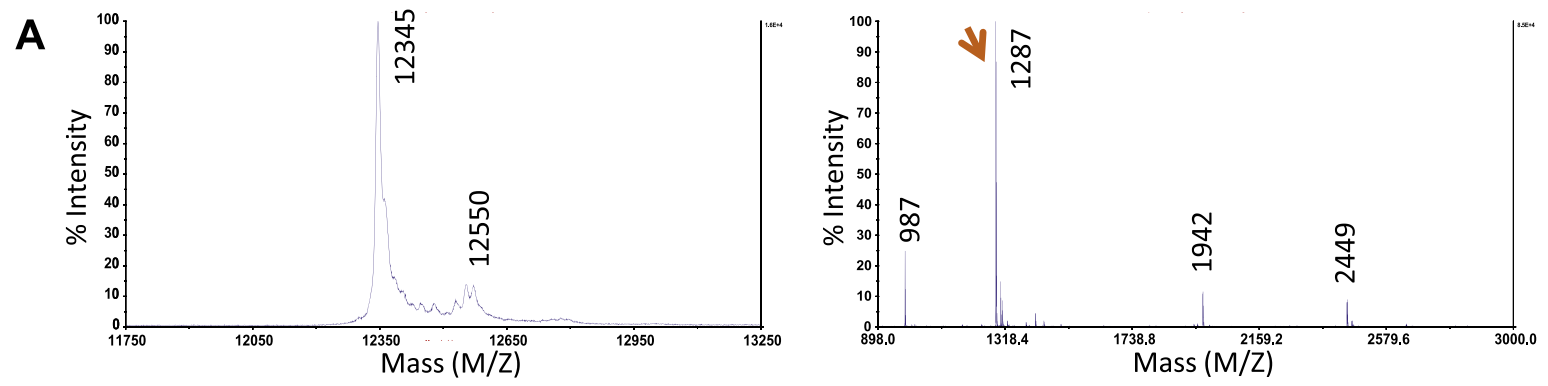

B

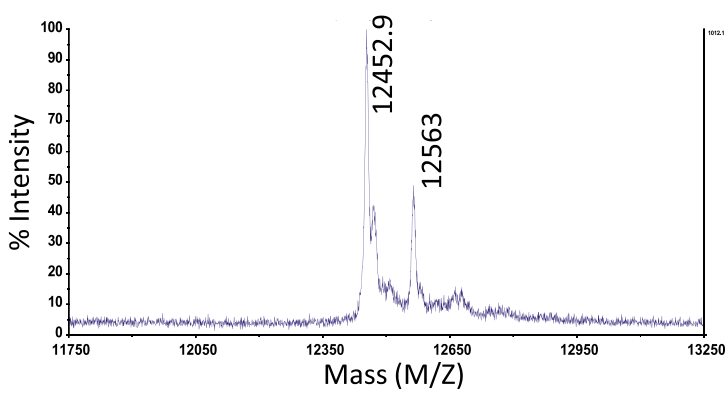

C

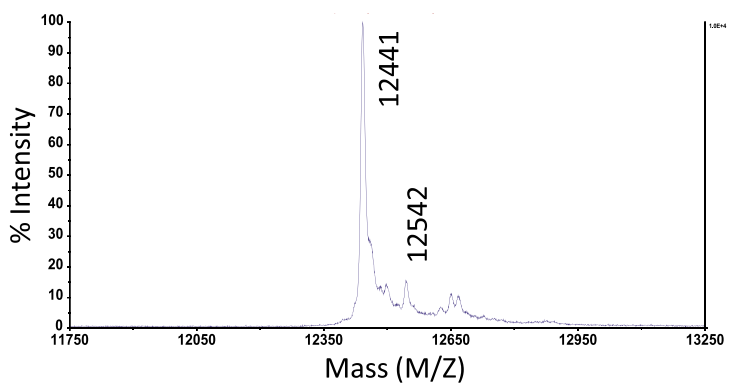

D

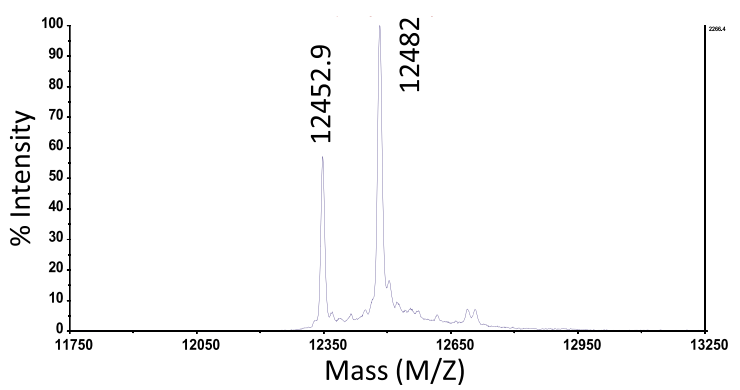

E

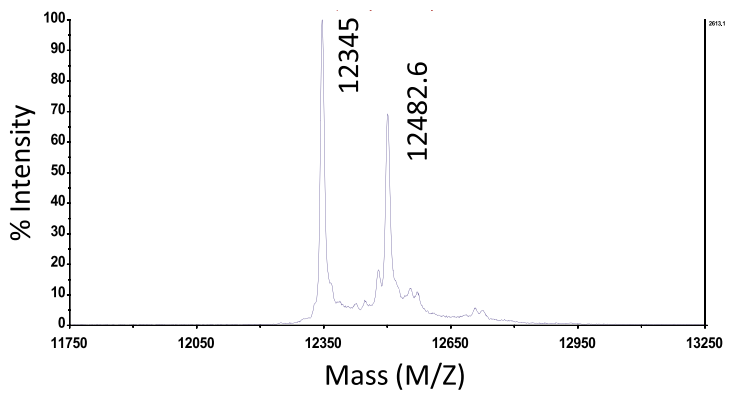

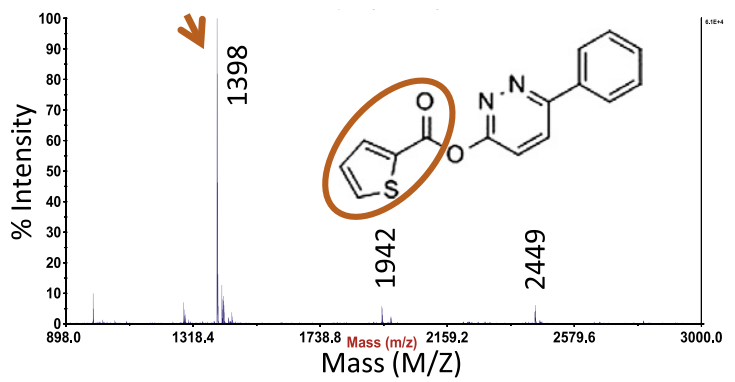
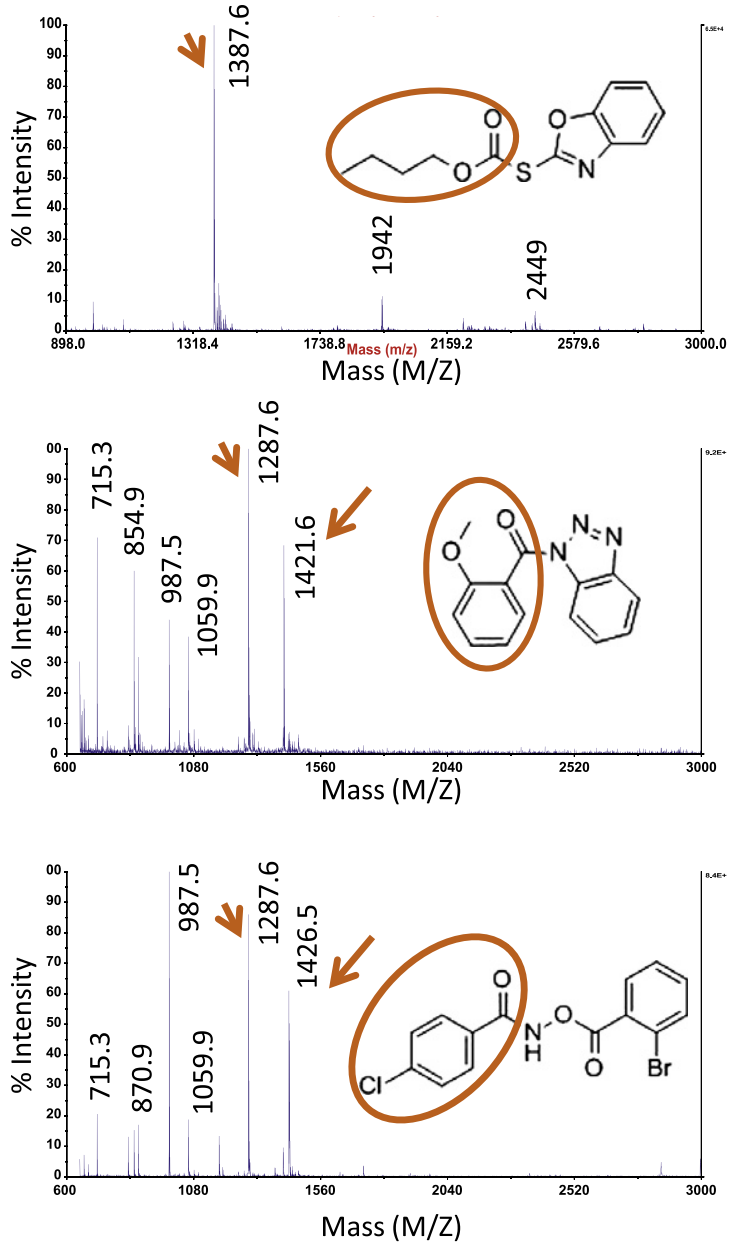

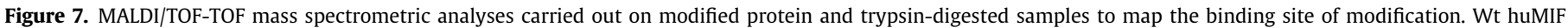

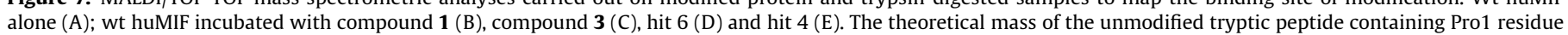
(PMFIVNTNVPR) is $1287.5 \mathrm{~g} / \mathrm{mol}$. The fragments of the compounds that remain covalently bound to Pro1 are shown encircled in red in the respective structures.

new MIF-CD74 interaction antagonists targeting the tautomerase active site, Cournia et al. performed virtual HTS by docking 2.1 million compounds, from Maybridge and Zinc databases that had been pre-filtered for properties based on Lipinski rules. Molecular 

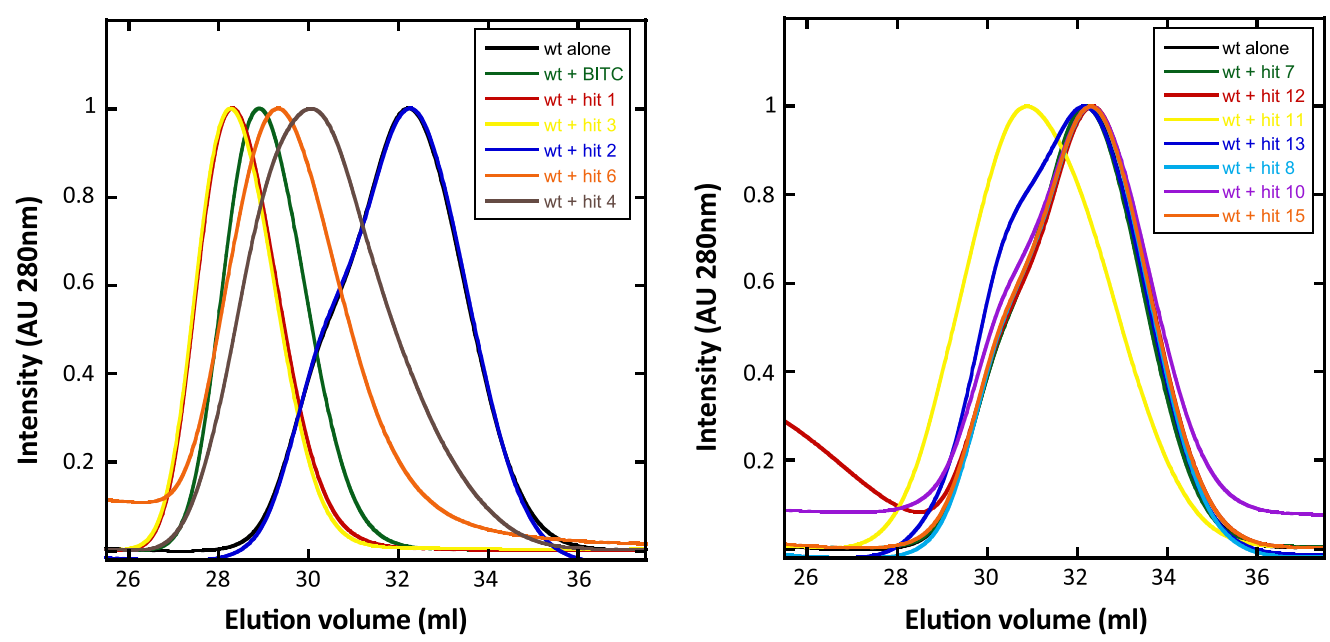

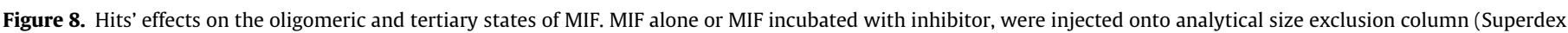

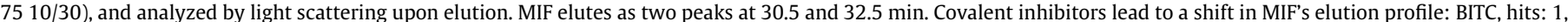
3, 6 and 4 lead to MIF's earlier elution as one peak; hits 11 and 13 lead to a change in the ratio Peak 1/Peak 2; all other inhibitors do not affect MIF's elution profile.

Table 4

Comparative evaluation of hit rates obtained for experimental HTS and by the in silico mixed methodology approach

\begin{tabular}{|c|c|c|c|c|c|c|c|c|c|}
\hline \multicolumn{2}{|l|}{ Protocol } & \multirow{2}{*}{$\begin{array}{l}\text { Library } \\
\text { Maybridge }\end{array}$} & \multirow{2}{*}{$\begin{array}{l}\text { Tested compounds } \\
14,400\end{array}$} & \multicolumn{2}{|l|}{ Hits } & \multicolumn{2}{|c|}{ Hit rate (\%) } & \multicolumn{2}{|c|}{ Enrichment factor } \\
\hline Experimental HTS & & & & 15 & & 0.105 & & 1 & \\
\hline Experimental HTS & & FDA & 1040 & 2 & & 0.192 & & 1.84 & \\
\hline In silico combined approach & MDS/FlexX & ChemBridge & 333 & 12 & 2 & 1.2 & 0.6 & 11.43 & 5.71 \\
\hline & MDS/Unity ${ }^{\circledR}$ & & 334 & & 4 & & 1.2 & & 11.43 \\
\hline & Surflex-Dock & & 333 & & 6 & & 1.8 & & 17.14 \\
\hline
\end{tabular}

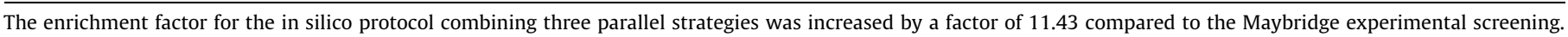

structures were docked and scored using the Glide docking program. Twenty six molecules were then selected by visual inspection of the 1200 top-scoring molecules, and eleven were confirmed as MIF tautomerase inhibitors effective in the micromolar range, with four compounds having $\mathrm{IC}_{50}$ as low as $0.5-5 \mu \mathrm{M}^{35}$ These results highlight the potential of the virtual screening approach for the discovery of novel and potent inhibitors of MIF catalytic and biological activities.

Until now, in silico-based approaches applied to MIF have used only one set of X-ray coordinates corresponding to a particular PDB entry. $^{34,35,44,57}$ Therefore, the dynamics of the MIF catalytic pocket in solution have not previously been taken into consideration. Nonetheless, NMR studies from our laboratory (Ouertatani-Sakouhi et al., 2010 and El-turk et al., 2010) and our MD simulations performed on MIF showed high dynamic fluctuations of the catalytic pocket (Fig. 3 and Table 1). We hypothesized that a better understanding of the flexibility of the catalytic site and how it contributes to ligands binding would facilitate computer assisted drug design of small molecule inhibitors of MIF activity and would enhance the success rates of in silico screening approaches. To test this hypothesis, we sought to develop an in silico protocol that allows better consideration of these parameters when screening large databases of chemical compounds for more potent and diverse inhibitors of MIF tautomerase activity. Towards this goal, we generated a novel and integrative virtual screening protocol that combines MD simulations aimed at modeling the protein's flexibility in solution, which we coupled to (i) docking with FlexX, or (ii) docking with FlexX and pharmacophoric filtering with Unity. Eight MD structures were selected based on their ability to retrieve all known inhibitors at the best tolerance; this tolerance $(0.8 \AA)$ represents a compromise between the ability of the pharmacophore to reject false positives and its capacity to retain as many true positives as possible (Fig. 2). This protocol was applied in par- allel with a standalone docking using the new version of Surflex software (2.4), and the X-ray structure of ligand-free MIF according to the PDB 1GD0 coordinates. Given the small chemical overlap between the compounds selected from these three approaches, we decided to select the top-ranked $1 \%$ identified by each method to increase the chances of obtaining highly potent hits.

An in silico HTS of Chembridge library was performed using the protocol described above. Finally, validation of the hits using tautomerase assays was then performed on the 333 best-scored molecules obtained by FlexX docking on MD structures, as well as on the 334 top-scored molecules obtained by FlexX and by the Unity pharmacophore, and the 333 top-scored Surflex docked compounds, for a total of 1000 compounds. This biochemical validation process resulted in the identification of 12 novel MIF inhibitors using FlexX + MDS (2), or FlexX + MDS + Unity (4) and Surflex (6). These results suggest that coupling these different approaches developing an integrative in silico protocol should facilitate the identification and discovery of novel classes of MIF inhibitors. Three hits were identified using Surflex Docking with $\mathrm{IC}_{50}$ values lower than $10 \mu \mathrm{M}$ (two hits with $\mathrm{IC}_{50}$ lower than $1 \mu \mathrm{M}$ : hits 1 and 3, Fig. $6 \mathrm{~A}$ and $\mathrm{B}$ ); these two were shown to be suicide inhibitors that act via covalent modification of the N-terminal catalytic residue Pro1, which is essential for MIF tautomerase activity. The activity of these compounds mirrors that of other Pro1 modifiers, $N$-acetyl-p-benzo-quinone imine (NAPQI, acetaminophens), ${ }^{33}$ 4-iodo-6-phenylpyrimidine (4-IPP) ${ }^{34}$ and isothiocyanates. ${ }^{52}$ Interestingly, studies from our group have shown that only catalytically active trimers undergo modification by these compounds at Pro1 in vitro and in the cell. ${ }^{52,58}$ These studies highlight the reactivity of the N-terminal proline, Pro1, and provide a basis of novel strategies for specific detection and quantification of catalytically active MIF and targeting of its catalytic activities. 
One additional inhibitor, $N$-phenyl- $N^{\prime}-1,3,4$-thiadiazol-2ylthiourea, $\left(\mathrm{IC}_{50}=300 \mathrm{nM}\right)$ was obtained from FlexX docking combined with pharmacophoric filtering on one of the eight MD structures (Fig. 6C). When the validation of the hits from the different approaches outlined above was carried out at higher compound concentration $(100 \mu \mathrm{M})$, we identified nine additional hits. Standalone docking with Surflex allowed the identification of five new hits with $\mathrm{IC}_{50}$ between 18.6 and $62.1 \mu \mathrm{M}$. Other active inhibitors (hits 11,12 , and 15 which have $\mathrm{IC}_{50}$ values of $>200,1.04$, and $64.2 \mu \mathrm{M}$, respectively) were obtained from docking + pharmacophore filtering combined with three MD simulation conformations.

Our hits can be clustered according to their chemical classes. Compounds 1, 3, 6, 10 and 11 belong to chemical classes that do not share any similarity with previously known inhibitors. Notably all of these hits have conjugated ring systems that fit inside the active site and are stabilized with aryl-aryl interactions provided by Tyr95 and/or Phe113. Moreover, the specificity of interaction is often provided by hydrogen bonding and salt bridge interactions involving at least two of the following residues: Lys32, Ile64, Pro1, Tyr95, Asn97. Compounds 2 and 12 belong to the 'phenylthiourea' chemical family. Figure $9 \mathrm{~B}$ illustrates the interaction of the computed best pose hit 2 within the catalytic site of MIF. As with previously known inhibitors, hydrogen bonds and salt bridges involving Ile64 and Lys32 seem to contribute significantly to the binding energy. Similarity searches carried out on the 1000 screened compounds identified five other molecules belonging to the same family. All five compounds were inactive and represent structural analogues of compound 2 (Supplementary Fig. S3). Unlike compound 2, the aromatic rings of these compounds contain additional substitutions on the phenyl or pyridine rings that appear to introduce sterical effects that distort the interactions observed with compound $\mathbf{1 2}$ and interfere with occupancy of the active site.

Compound $\mathbf{4}$ is predicted to bind to the enzymatic pocket in a manner similar to the known inhibitors. Edge-to-face aryl-aryl interactions, as well as hydrogen bonds involving Lys32, Ile64 and Pro1, participate in the stabilization of compound $\mathbf{4}$ within the pocket. Two inactive close structural analogues that differ with respect to the substitution of one of the phenyl rings were identified among the 1000 compounds screened. Changing the type of halogen atoms or its substitution position, as well as the incorporation of additional substitutions (e.g., $\mathrm{Cl}$ instead of $\mathrm{Br}$ ) appears to be sufficient to abolish the activity of compound 4 , highlighting the specificity with which this inhibitor interacts within the active site (Supplementary Fig. S3).

Despite the fact that most of inhibitors discovered in this study do not share structural similarities with known inhibitors of MIF, our in silico protocol identified two coumarin derivatives (compounds 8 and 15), one of which was previously identified as a potent tautomerase inhibitor ${ }^{44}$ and co-crystallized with MIF (PDB code: 1GCZ). Orita et al. ${ }^{44}$ demonstrated that compound 8 has a $K_{\mathrm{i}}$ of $4 \mu \mathrm{M}$; whereas our studies showed that it inhibits MIF with an $\mathrm{IC}_{50}$ of $45.2 \mu \mathrm{M}$. Plausible explanations for this discrepancy are the fact that our $\mathrm{IC}_{50}$ calculations were based on end point absorbance (i.e., absorbance of the substrate after 2 min reaction with the enzyme), whereas $K_{\mathrm{i}}$ values in Orita et al. were calculated from the initial velocity of reaction of the substrate with the enzyme. Moreover, our experimental design is based on the use of higher amounts of substrate and enzyme (MIF, $500 \mathrm{nM}$ versus $320 \mathrm{nM}$ in Orita et al.'s study; substrate: $533 \mu \mathrm{M}$ versus 250-500 $\mu \mathrm{M}$ used by Orita et al. $^{44}$ ). Compound 15, which is also a coumarin derivative and has not been reported previously, showed a higher $\mathrm{IC}_{50}(64.2 \mu \mathrm{M})$. Analysis of the 1000 screened compounds demonstrated the existence of nine other inactive coumarin analogues (Supplementary Fig. S3). Examination of the computed best poses of hits 8 and 15 demonstrated the importance of the hydroxyl at position 7, which interacts with Asn97 through hydrogen bonding and stabilizes the molecule within the active site (Fig. 9), consistent with previous studies by Orita et al. ${ }^{44}$

The inhibition curves shown in Figure 5 are unusually steep for some compounds (e.g., 1, 2, 3, 4, 5, 6, 9, and 11); this could be indicative of compound aggregation at particular concentrations, resulting in false positive inhibition. To examine this possibility, we performed the concentration-dependant inhibition assays with the 15 initially confirmed hits, and determined that hits 5, 9, and 14 are most likely promiscuous aggregators, and were discarded from the enrichment factor calculations. Inhibition of MIF by these molecules was abolished in the presence of $1 \%$ of detergents (Fig. 5). The basis underlying the sharp inhibition curves has been discussed extensively by Shoichet ${ }^{59}$; in our case the steepness of the curve for some of the validated inhibitors could be explained by the facts that MIF has three catalytic sites and/or the high enzyme concentrations used in our assays (500 nM). This concentration of MIF was selected to reduce the effects of enzyme adsorption to the walls of microtiter plates. This may lead to the effects observed in the case of high [enzyme] $/ K_{\mathrm{d}}$ ratios. We believe that this is the basis for the high Hill coefficients we observed for the compounds (hits $1,2,3,4,6$, and 11 ) which we validated as non-aggregating inhibitors.

Molecular docking experiments predicted that the inhibitors 1 , 3, 4 and 6 interact with the active site of MIF. Experimentally they proved to act as suicide inhibitors, thereby casting doubts about counting them as computational successes. However, we showed that all four inhibitors only react with MIF's catalytic Pro1 residue, which requires that they fit and adopt the correct orientation within the catalytic pocket of MIF. It is also plausible that the high reactivity of Pro1 may be sufficient for covalent modification, although the efficiency of covalent modification suggest that both of these
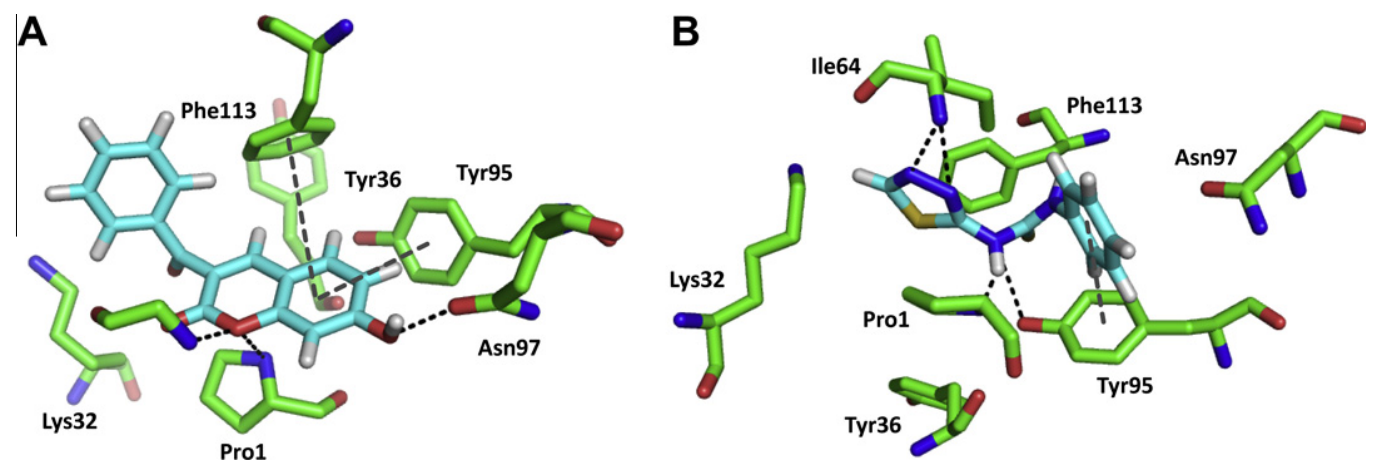

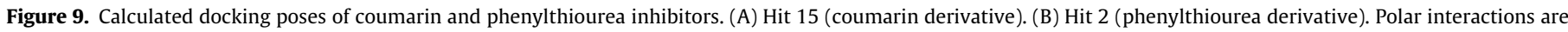
shown as black dotted lines; aryl-aryl contacts are pictured in gray. 
factors contribute to the potency of these inhibitors. Furthermore, uncertainties about their specificity were ruled out experimentally. We showed that the four inhibitors failed to modify other proteins, suggesting that they modify MIF in a specific manner. (Supplementary Fig. S4C-F). We chose proteins that contain potentially nucleophilic groups such as cysteines and lysines, which would therefore have been modified by hits $1,3,4$, and 6 if they were not specific for MIF. The finding that hits 1 and 3 covalently bind to 4-oxalocrotonate tautomerase (Supplementary Fig. S4B) does not dismiss them. As we have shown that these molecules react with MIF's Pro1 residue (Fig. 7), it is not surprising that these compounds modify 4-OT. Like MIF, these enzymes are homotrimers (4OT is a trimer of homodimers) and belong to the tautomerase super family characterized by a $\beta-\alpha-\beta$ motif and a catalytic amino-terminal proline. ${ }^{31}$ Mutation of the catalytic Pro1 residue in MIF, 4-OT, and 5-CHMI abolishes the tautomerase activity of these enzymes. ${ }^{31,32}$ Together, all these data suggest that the covalent hits $1,3,4$ and 6 we present here are novel, specific irreversible modulators of MIF's tautomerase function.

\section{Conclusion}

Using our integrative virtual screening approach we identified and validated 12 novel inhibitors of MIF tautomerase activity. The chemical diversity and mode of action of these compounds suggest that they could be used as molecular probes to elucidate the functions and biology of MIF and as potential lead candidates in drug developments of anti-MIF drugs. Taken together, these findings confirm the importance of MD simulations for the virtual screening, since FlexX used on the X-ray coordinates of MIF alone would not have identified these inhibitors. Moreover, the fact that the compounds obtained using the three different approaches belong to different chemical classes and exhibit different modes of action underscores the importance of coupling different computational approaches for optimizing screening protocols. The combination of two docking softwares with molecular dynamic simulations and pharmacophore filtering demonstrates one more time the power of in silico procedures as a complementary approach in the discovery of novel inhibitors of enzymes and other biological systems. Combining the eight structures obtained from MD simulations with docking with either FlexX or Surflex and pharmacophore filtering with Unity can now be applied to very large databases of small compounds in the hope to discovering new scaffolds inhibiting MIF activity.

\section{Methods}

\subsection{Molecular dynamics simulations}

All MD simulations were performed in explicit solvent with periodic conditions with the CHARMM package version 33b1 and CHARMM force field version 22 with CMAP correction. ${ }^{45-48}$ The initial coordinates were taken from the ligand-free crystal structure solved at a resolution of $1.5 \AA$ (PDB ID: $1 \mathrm{GDO}^{44}$ ). Non-protein derivatives such as citric acid and $\mathrm{SO}_{4}{ }^{2-}$ ions were not taken into account. C-terminal His-tag residues were removed from the PDB file. Orientation of the side chains of Asn, Gln, and His residues were checked using the in-house VMD plugin checksidechains (http://wwws.ks.uiuc.edu/Research/vmd/plugins/) and using the WHAT IF web interface (http://swift.cmbi.kun.nl/). Ionization states of titratable residues were determined according to empirical $\mathrm{p} K_{\mathrm{a}}$ and Poisson-Boltzmann calculations. Residues His40 and His62 were assigned HSE types. All hydrogens were considered explicitly in the calculation. Coordinates of missing hydrogen atoms were added using the hbuild algorithm in CHARMM. To improve conformational sampling, eight independent simulations were carried out using different initial conditions, which are summarized in Supplementary Table $S 1$. The system was solvated with a pre-equilibrated solvation box (edge length around $76 \AA$ ) consisting of TIP3P water molecules where periodic boundary conditions were applied. Crystallographic water molecules were included in the initial model in four of the eight conditions; all crystal water molecules were removed in the remaining conditions (Supplementary Table S1). Chloride and sodium ions were added to ensure a neutral system. Unfavorable contacts were removed by a short energy minimization with conjugate gradient and ABNR. Electrostatic interactions were treated using the particle-mesh Ewald summation method, and we used the switch function for the van der Waals energy interactions with cuton, cutoff and cutnb values of 9, 11 and $13 \AA$, respectively. The Shake algorithm ${ }^{60}$ was applied to all hydrogen-containing bonds and a $1 \mathrm{fs}$ integration step was used. The system was heated gradually to the desired temperature ( 300 or $333 \mathrm{~K}$ ), followed by a further equilibration step (150 ps). During these two early steps, harmonic constraints were applied to protein heavy atoms. The constraint harmonic constant $(k)$ was equal to 1 and $0.1 \mathrm{kcal} / \mathrm{mol} / \AA^{2}$ for the backbone and side chains, respectively. The production phase was performed without any constraints. Snapshots of the coordinates were saved every 500 steps ( 0.5 ps) leading to about 4000 instantaneous conformations for each trajectory. A typical 2 ns simulation required about three weeks CPU time on a $3.5 \mathrm{GHz}$ processor. Trajectories were analyzed using a combination of CHARMM, in house Perl or Matlab scripts, and VMD. Overall $<$ RMSD $>$ variations were computed with CHARMM after superimposition of the CA atoms.

\subsection{Ligand library}

Virtual screening methods were applied to the NINDS library of FDA-approved molecules (1040 compounds), the HitFinder diverse subset of the Maybridge database (14,400 compounds), and the Chembridge library ( $\sim 500,000$ compounds obtained from ChemBridge Corporation). The last library, in 2D Structure Definition File format (SDF), was transformed into a 3D database using the CONCORD software (implemented in Tripos' Sybyl 8.0). Default parameters were employed for the conversion and all anilinic nitrogen were designed in a planar conformation. The library was then filtered using the Tripos SELECTOR module to retain only structures with 30 or fewer non-hydrogen atoms, resulting in a final dataset of 33,900 compounds. Positive control ligands were obtained from MIF X-ray structures where the protein had been co-crystallized with substrate (PDB code 1CA7) or with tautomerase inhibitors (PDB codes 1GCZ, 1LJT, 1MFI, and 200Z). Coordinates of all ligands were then refined using the energy minimization module of Sybyl (MMFF94 force field).

\subsection{Docking}

Protein-ligand complexes were simulated using FlexX version 3.0.2 (BioSolveIT, Germany), and Surflex version 2.4 implemented in Sybyl 8.0 from Tripos, Inc. ${ }^{61}$ The MIF residues that defined the active site in our docking studies are: (Subunit C) Pro1, Met2, Thr30, Gly31, Lys32, Pro33, Pro34, Tyr36, Ile37, Ala38, Ser60, Leu61, His62, Ser63, Ile64, Gly65, Lys66, Ile67, Asp100, Met101, Ala103, Val106, Gly107, Trp108, Phe113, and (Subunit B), Phe49, Gly50, Val94, Tyr95, Ile96, Asn97. Formal charge assignments for both receptors and ligands were made using the default parameters of FlexX, except for Pro1 of MIF, which is assumed to be neutral under physiological conditions because of its $\mathrm{p} K_{\mathrm{a}}$ of $5.6-6$. $^{62}$ For each compound docked, we saved the 50 best poses according to FlexX scoring function (F-Score ${ }^{63}$ ). Additionally, each pose was evaluated using functions from the CScore module of Sybyl. ${ }^{64}$ This consensus score (CScore) integrates a number of popular scoring 
functions to rank ligand's affinity for the active site of the receptor. D_score and G_score are two Force-Field derived scoring functions. D_score, derived from DOCK score in the FlexX implementation, uses steric and electrostatic terms based on the AMBER ForceField, ${ }^{65}$ whereas G_score, derived from GOLD score in the FlexX implementation, is a sum of the hydrogen bonding stabilization energy (calculated from van der Waals energy for the ligand and conformers) and a pair wise dispersion potential between ligand and protein that describes the hydrophobic binding energy. ${ }^{66,67} \mathrm{~A}$ knowledge-based scoring function, PMF score, exploits structural information of known complexes and converts it into distancedependent Helmholtz free energies. ${ }^{68}$ Finally, two empirical scoring functions were implemented: ChemScore consists of a term that estimates lipophilic contact energy, a metal-ligand binding contribution, an empirical form for hydrogen bonds, and a penalty for ligand flexibility, ${ }^{69}$ whereas FlexX score considers the number of rotatable bonds in the ligand, hydrogen bond interaction, ion pairing, aromatic interactions, and lipophilic contact energy. ${ }^{70}$ The final choice of the best scoring function was optimized on the basis of the docking results of the positive control ligands (known X-ray structures). The Surflex docking was carried out on MIF apo-structure (PDB code 1GD0) using the amino acids described above to generate the Surflex protomol (Surflex active site definition) with standard parameters (threshold 0.5 , no bloat). One third of randomly picked compounds within the ChemBridge filtered database (11,300 compounds) were screened using the Geom $\mathrm{X}$ option from Surflex-Dock version 2.4. According to Surflex-Dock scoring function, the 333 best-docked compounds were selected for further analysis (top 3\%).

\subsection{Pharmacophore-based search}

A pharmacophore model of MIF's active site was built with the UNITY component of Sybyl 8.0. Spatial constraints were built for the following residues: (1) Acceptor sites: Pro1-A (N atom lone pair), Tyr36-A (carbonyl O), Ile64-A (carbonyl O), Asn97-B (side chain amide), and Met101-B (carbonyl O); (2) Donor sites: Lys32A (side chain ammonium), Ala38-A (amide N), His62-A (amine N), and Ile64-A (amide N). Ligand structures were selected as hits if they presented at least two donor atoms matching the receptor's 'Acceptor site' constraints and one acceptor atom fitting into a 'Donor site' query. The tolerances of the spatial pharmacophoric constraints (i.e., the allowed deviation from a perfect match) were optimized over a range of 0.5-2.0 A, using known X-ray structures.

\section{Experimental section}

\subsection{Chemicals}

Compounds were purchased from ChemBridge Corporation (www.chembridge.com). Miscellaneous chemicals were purchased from Sigma-Aldrich Chemicals, unless otherwise stated and are of the highest grade commercially available. Trypsin was purchased from Promega.

\subsection{Expression and purification of MIF}

Wild type human MIF (wt huMIF) was expressed and purified as described previously. ${ }^{28}$

\subsection{Synthesis of the D-dopachrome methyl ester substrate for keto-enol tautomerase assay}

The D-dopachrome methyl ester was freshly prepared by addition of periodate $\left(\mathrm{NaIO}_{4}\right)(8 \mathrm{mM})$ to L-3,4-dihydroxyphenylalanine methyl ester $(4 \mathrm{mM})$ for $5 \mathrm{~min}$ at room temperature and then placed directly on ice.

\subsection{Multi-well plate in vitro screening of the 1000 best-scored molecules identified by virtual HTS}

To validate our in silico screening protocol, we constructed a small library of 1000 compounds consisting of (i) the 333 highest ranking molecules obtained from docking with Surflex (Docking standalone with the geomX option toggled on); (ii) the 333 highest ranking molecules obtained from Docking with FlexX using different structures of the catalytic site (Docking + MDS); and (iii) the 334 highest ranking molecules obtained upon Docking with FlexX + Unity Pharmacophore filtering (PF) on the different structures of the catalytic site as well (Docking + MDS + PF). The compounds were dissolved to prepare a stock solution of $10 \mathrm{mM}$ in $100 \%$ DMSO, in three 384-well plates (384-well Corning clear plates). To perform the tautomerase assay in a highthroughput format, wt huMIF (final concentration: $500 \mathrm{nM}$ ) was added to 384-well plates (Nunc, Black wall, clear bottom) containing potassium phosphate buffer $(50 \mathrm{mM})$, EDTA $(0.5 \mathrm{mM})$, at $\mathrm{pH} 6$, and the compound of interest at a final concentration of $10 \mu \mathrm{M}$ or $100 \mu \mathrm{M}$ (final percentage of DMSO lower than $1.5 \%$ ). Wt huMIF and the compound were mixed and incubated for $30 \mathrm{~min}$ at $\mathrm{rt}$. The reaction was initiated by adding freshly prepared D-dopachrome methyl ester substrate (prepared as described above). The plates were centrifuged for 2 min at $2000 \mathrm{rpm}$ to remove air bubbles and the absorbance was measured at $475 \mathrm{~nm}$ using a Tecan Safire II reader. BITC, which was previously shown to completely inhibit MIF at $10 \mu \mathrm{M},{ }^{52}$ was used as a positive control. The screening assay was carried out in triplicate. Inhibitors' potencies were measured by recording the absorbance at the end point, that is, absorbance of the substrate 2 min after initiation of reaction with the enzyme.

\subsection{Validation and $\mathrm{IC}_{50}$ measurements of in vitro screening hits}

Primary hits obtained as described above were subjected to a validation assay in 384-well plates under the same conditions as for HTS. Buffer, enzyme and substrate solutions were added to the wells using an EL406 dispenser robot (BioTek Instruments). The validation assay was performed in five replicates. $\mathrm{IC}_{50}$ calculations were done using the same microtiter plate-based assay and were carried out in triplicate. Compounds were incubated with $500 \mathrm{nM}$ wt huMIF for $2 \mathrm{~h}$ at room temperature, at concentrations ranging from $24 \mathrm{nM}$ to $50 \mu \mathrm{M}$ (hits $1-5$ ), or 98 to $200 \mu \mathrm{M}$ (hits $6-15$ ), in absence or presence of $0.1 \% \mathrm{v} / \mathrm{v}$ Triton $\mathrm{X}-100$ in both buffer and enzyme solutions, in order to check for compound aggregation. Mixing was carefully done below the surface of the liquid in order to minimize the formation of bubbles. Relative inhibitions were determined from absorbance measurement at $475 \mathrm{~nm}$ after 2 min of enzymatic reaction. $\mathrm{IC}_{50}$ values were determined by nonlinear regression in Matlab 7.5 (MathWorks).

\subsection{MIF aggregation studies}

To determine if the validated hits induce the aggregation of MIF, wt huMIF $(\sim 15 \mu \mathrm{M})$ was incubated in $1 \mathrm{X}$ PBS with each compound at concentrations ranging from $15 \mu \mathrm{M}$ to the $\mathrm{IC}_{50}$ value (if higher than $15 \mu \mathrm{M}$ ), for $1 \mathrm{~h}$ at room temperature. The MIF-compound mixture was then filtered with a $0.2 \mu \mathrm{m}$ centrifugal filter (Millipore) and the retentate was solubilized in $0.1 \%$ SDS. Both the supernatant and the retentate were analyzed on a 15\% SDS-PAGE. Two controls of protein incubated without compound, or with ebselen (a molecule that induces MIF aggregation ${ }^{50}$ ) were used. 


\subsection{MALDI/TOF-TOF MS molecular mass measurements for examining possible protein modifications}

To determine whether any of the identified inhibitors covalently modifies MIF, mass spectrometric analysis of wt huMIF incubated with each inhibitor was performed by matrix-assisted laser desorption ionization (MALDI) MS using a linear positive ion mode on ABI 4700 (PCF Lausanne). Linear mode calibration was applied with a single point using human $\alpha$-synuclein $\left(\mathrm{MH}^{+}:\right.$14461.19). Sample preparation: $10 \mu \mathrm{M}$ of huMIF protein was incubated for $1 \mathrm{~h}$ at rt with $10 \mu \mathrm{M}$ of inhibitor; then $1 \mu \mathrm{L}$ of MIF-inhibitor mixture was mixed with $4 \mu \mathrm{L}$ of matrix solution, which was prepared by dissolving $14 \mathrm{mg}$ of sinapinic acid (SA) in $0.1 \% \mathrm{TFA} /$ acetonitrile mixture $(1: 1)$. One microliter of the sample-matrix mixture was then deposited on the MALDI plate.

\subsection{Protein digestion analysis to identify the residue(s) modified by the suicide compounds}

To identify the exact residues being covalently modified by MIF inhibitors, $200 \mu \mathrm{L}$ of wt huMIF $(30 \mu \mathrm{M})$ is incubated at rt for $1 \mathrm{~h}$ with $30 \mu \mathrm{M}$ of the covalent inhibitor (e.g., hits 1, 3, 4 and 6). Digestion with trypsin (1:50 protein weight) was then performed over night at $37^{\circ} \mathrm{C}$, with and quenched with $1 \mu \mathrm{L}$ of formic acid. Digested samples were analyzed by MALDI MS/MS on a 4700 MALDI/TOF-TOF instrument, as described in the previous paragraph.

\subsection{Effects of compounds on the tertiary and quaternary struc- ture of MIF}

To determine whether the MIF inhibitors identified in our screen act by inducing changes in the tertiary and/or quaternary structure of MIF, MIF-inhibitor mixtures were analyzed by static light scattering coupled with size exclusion chromatography. Measurements were carried out at room temperature on a DAWN HELEOS multi-angle light scattering detector (Wyatt Technology Corp, Santa Barbara CA) online with a size exclusion chromatography column (Superdex 75 10/300). The system is also equipped with $\mathrm{UV}$ and refractive index detector. The buffer used for equilibration of the gel filtration column was $20 \mathrm{mM}$ Tris, $150 \mathrm{mM} \mathrm{NaCl}, 0.05 \%$ $\mathrm{w} / \mathrm{v} \mathrm{NaN}$, pH 7.4. A volume of $100 \mu \mathrm{L}$ of MIF samples $(20-30 \mu \mathrm{M}$ in $1 \mathrm{X}$ PBS), incubated with $10 \mu \mathrm{M}$ of inhibitors for $1 \mathrm{~h}$ at $\mathrm{rt}$, was injected onto the column and eluted at a flow rate of $0.4 \mathrm{~mL} / \mathrm{min}$. Absolute MWs were determined using ASTRA version 5.3 from Wyatt Technologies.

\section{Acknowledgments}

Financial support for these studies was provided by the Swiss Federal Institute of Technology Lausanne (HAL, FE, HO), and a grant from the Swiss National Science Foundation (HAL, FE-310000110027). We thank Dr. Marc Moniatte, Mr. Diego Chiappe, Mr. Jonathan Paz Montoya and Mr. Jérome Vialaret from the proteomic facility (http://pcf.epfl.ch) for their assistance and advices. We also thank Dr. Gerardo Turcatti, Dr. Damiano Banfi, Dr. Marc Chambon and M. Miquel Busquets Lopez from the screening facility (http:// bsf.epfl.ch) for their help to perform the in vitro screening and validation of the hits. We finally thank Prof. Brian Shoichet and Mrs. Allison Doak from the University of California, San Francisco for their very kind assistance about the detergent-based assay.

\section{Supplementary data}

Supplementary data (includes two tables describing the $8 \mathrm{MD}$ simulation conditions used in the in silico study, and representing the distances between key residues to select structures where the catalytic pocket has a suitable conformation for ligand docking; as well as the mass spectrometry data for the incubation of the covalent hits with five different proteins) associated with this article can be found, in the online version, at doi:10.1016/ j.bmc.2010.05.010.

\section{References and notes}

1. Bloom, B. R.; Bennett, B. Science 1966, 153, 80

2. David, J. R. Proc. Natl. Acad. Sci. U.S.A. 1966, 56, 72

3. Weiser, W. Y.; Pozzi, L. M.; David, J. R. J. Immunol. 1991, 147, 2006.

4. Pozzi, L. A.; Weiser, W. Y. Cell Immunol. 1992, 145, 372.

5. Bernhagen, J.; Calandra, T.; Mitchell, R. A.; Martin, S. B.; Tracey, K. J.; Voelter, W.; Manogue, K. R.; Cerami, A.; Bucala, R. Nature 1993, 365, 756.

6. Calandra, T.; Echtenacher, B.; Roy, D. L.; Pugin, J.; Metz, C. N.; Hultner, L.; Heumann, D.; Mannel, D.; Bucala, R.; Glauser, M. P. Nat. Med. 2000, 6, 164

7. Onodera, S.; Kaneda, K.; Mizue, Y.; Koyama, Y.; Fujinaga, M.; Nishihira, J. J. Biol. Chem. 2000, 275, 444

8. Morand, E. F.; Leech, M.; Bernhagen, J. Nat. Rev. Drug Disc. 2006, 5, 399.

9. Santos, L. L.; Morand, E. F. Wien. Med. Wochenschr. 2006, 156, 11.

10. Bernhagen, J.; Mitchell, R. A.; Calandra, T.; Voelter, W.; Cerami, A.; Bucala, R. Biochemistry 1994, 33, 14144.

11. Calandra, T.; Bernhagen, J.; Mitchell, R. A.; Bucala, R. J. Exp. Med. 1994, 179, 1895.

12. Herriott, M.; Jiang, H.; Stewart, C.; Fast, D.; Leu, R. J. Immunol. 1993, 150, 4524.

13. Onodera, S.; Suzuki, K.; Matsuno, T.; Kaneda, K.; Takagi, M.; Nishihira, J. Immunology 1997, 92, 131.

14. Bacher, M.; Metz, C. N.; Calandra, T.; Mayer, K.; Chesney, J.; Lohoff, M.; Gemsa, D.; Donnelly, T.; Bucala, R. Proc. Natl. Acad. Sci. U.S.A. 1996, 93, 7849.

15. Yang, Y.; Degranpré, P.; Kharfi, A.; Akoum, A. J. Clin. Endocrinol. Metab. 2000, 85, 4721.

16. Mitchell, R. A.; Metz, C. N.; Peng, T.; Bucala, R. J. Biol. Chem. 1999, 274, 18100.

17. Lolis, E. Curr. Opin. Pharmacol. 2001, 1, 662.

18. Van Molle, W.; Libert, C. Eur. J. Immunol. 2005, 35, 3396.

19. Awandare, G. A.; Martinson, J. J.; Were, T.; Ouma, C.; Davenport, G. C.; Ong'echa, J. M.; Wang, W.; Leng, L.; Ferrell, R. E.; Bucala, R.; Perkins, D. J. J. Infect Dis. 2009, 200, 629.

20. Bando, H.; Matsumoto, G.; Bando, M.; Muta, M.; Ogawa, T.; Funata, N.; Nishihira, J.; Koike, M.; Toi, M. Jpn. J. Cancer Res. 2002, 93, 389.

21. Bucala, R.; Donnelly, S. C. Immunity 2007, 26, 281.

22. Calandra, T.; Roger, T. Nat. Rev. Immunol. 2003, 3, 791.

23. Cvetkovic, I.; Stosic-Grujicic, S. Int. Immunopharmacol. 2006, 6, 1527.

24. Lue, H.; Kleemann, R.; Calandra, T.; Roger, T.; Bernhagen, J. Microbes Infect. 2002, 4, 449.

25. Sun, H. W.; Swope, M.; Cinquina, C.; Bedarkar, S.; Bernhagen, J.; Bucala, R.; Lolis, E. Protein Eng. 1996, 9, 631.

26. Sun, H. W.; Bernhagen, J.; Bucala, R.; Lolis, E. Proc. Natl. Acad. Sci. U.S.A. 1996, 93, 5191.

27. Muhlhahn, P.; Bernhagen, J.; Czisch, M.; Georgescu, J.; Renner, C.; Ross, A.; Bucala, R.; Holak, T. A. Protein Sci. 1996, 5, 2095.

28. El-Turk, F.; Cascella, M.; Ouertatani-Sakouhi, H.; Narayanan, R. L.; Leng, L.; Bucala, R.; Zweckstetter, M.; Rothlisberger, U.; Lashuel, H. A. Biochemistry 2008, 47, 10740.

29. Lubetsky, J. B.; Swope, M.; Dealwis, C.; Blake, P.; Lolis, E. Biochemistry 1999, 38 , 7346.

30. Swope, M.; Sun, H.; Blake, P.; Lolis, E. EMBO J. 1998, 17, 3534

31. Poelarends, G. J.; Veetil, V. P.; Whitman, C. P. Cell Mol. Life Sci. 2008, 65, 3606.

32. Whitman, C. P. Arch. Biochem. Biophys. 2002, 402, 1.

33. Senter, P. D.; Al-Abed, Y.; Metz, C. N.; Benigni, F.; Mitchell, R. A.; Chesney, J.; Han, J.; Gartner, C. G.; Nelson, S. D.; Todaro, G. J.; Bucala, R. Proc. Natl. Acad. Sci. U.S.A. 2002, 99, 144.

34. Winner, M.; Meier, J.; Zierow, S.; Rendon, B. E.; Crichlow, G. V.; Riggs, R.; Bucala, R.; Leng, L.; Smith, N.; Lolis, E.; Trent, J. O.; Mitchell, R. A. Cancer Res. 2008, 68, 7253.

35. Cournia, Z.; Leng, L.; Gandavadi, S.; Du, X.; Bucala, R.; Jorgensen, W. L. J. Med. Chem. 2009, 52, 416.

36. Al-Abed, Y.; Dabideen, D.; Aljabari, B.; Valster, A.; Messmer, D.; Ochani, M.; Tanovic, M.; Ochani, K.; Bacher, M.; Nicoletti, F.; Metz, C.; Pavlov, V. A.; Miller, E. J.; Tracey, K. J. J. Biol. Chem. 2005, 280, 36541.

37. Rendon, B. E.; Roger, T.; Teneng, I.; Zhao, M.; Al-Abed, Y.; Calandra, T.; Mitchell, R. A. J. Biol. Chem. 2007, 282, 29910.

38. Meyer-Siegler, K. L.; Iczkowski, K. A.; Leng, L.; Bucala, R.; Vera, P. L. J. Immunol. 2006, $177,8730$.

39. Choi, H.; Lee, Y.; Lee, S.; Hong, S.; Hahm, D.; Choi, S.; Yang, H.; Yoo, M.; Kim, K. Arthritis Res. Ther. 2009, 11.

40. So, A.; Chamot, A.; Peclat, V.; Gerster, J. Rheumatology 1999, 38, 407.

41. Lubetsky, J. B.; Dios, A.; Han, J.; Aljabari, B.; Ruzsicska, B.; Mitchell, R.; Lolis, E.; Al-Abed, Y. J. Biol. Chem. 2002, 277, 24976.

42. Taylor, A. B.; Johnson, W. H., Jr.; Czerwinski, R. M.; Li, H. S.; Hackert, M. L.; Whitman, C. P. Biochemistry 1999, 38, 7444

43. Zhuang, S.; Zou, J.; Jiang, Y.; Mao, X.; Zhang, B.; Liu, H.; Yu, Q. J. Med. Chem. 2005, 48, 7208 . 
44. Orita, M.; Yamamoto, S.; Katayama, N.; Aoki, M.; Takayama, K.; Yamagiwa, Y.; Seki, N.; Suzuki, H.; Kurihara, H.; Sakashita, H.; Takeuchi, M.; Fujita, S.; Yamada, T.; Tanaka, A. J. Med. Chem. 2001, 44, 540.

45. MacKerell, A. D. J.; Bashford, D.; Bellott, M.; Dunbrack, R. L. J.; Evanseck, J. D.; Field, M. J.; Fischer, S.; Gao, J.; Guo, H.; Ha, S.; Joseph-McCarthy, D.; Kuchnir, L.; Kuczera, K.; Lau, F. T. K.; Mattos, C.; Michnick, S.; Ngo, T.; Nguyen, D. T.; Prodhom, B.; Reiher, W. E. I.; Roux, B.; Schlenkrich, M.; Smith, J. C.; Stote, R.; Straub, J.; Watanabe, M.; Wiórkiewicz-Kuczera, J.; Yin, D.; Karplus, M. J. Phys. Chem. 1998, 102, 3586

46. Mackerell, A. D., Jr. J. Comput. Chem. 2004, 25, 1584.

47. Mackerell, A. D., Jr.; Feig, M.; Brooks, C. L., 3rd. J. Comput. Chem. 2004, 25, 1400.

48. Buck, M.; Bouguet-Bonnet, S.; Pastor, R. W.; MacKerell, A. D., Jr. Biophys. J. 2006, 90, L36.

49. Crichlow, G. V.; Cheng, K. F.; Dabideen, D.; Ochani, M.; Aljabari, B.; Pavlov, V. A.; Miller, E. J.; Lolis, E.; Al-Abed, Y. J. Biol. Chem. 2007, 282, 23089.

50. Ouertatani-Sakouhi, H.; El-Turk, F.; Fauvet, B.; Cho, M. K.; Le Roy, D.; Dewor, M.; Roger, T.; Bernhagen, J.; Calandra, T.; Zweckstetter, M.; Lashuel, H. A. J. Biol.Chem. 2010, submitted for publication.

51. Feng, B. Y.; Shoichet, B. K. Nat. Protoc. 2006, 1, 550.

52. Ouertatani-Sakouhi, H.; El-Turk, F.; Fauvet, B.; Roger, T.; Le Roy, D.; Karpinar, D. P.; Leng, L.; Bucala, R.; Zweckstetter, M.; Calandra, T.; Lashuel, H. A. Biochemistry 2009, 48, 9858.

53. Stivers, J. T.; Abeygunawardana, C.; Mildvan, A. S.; Hajipour, G.; Whitman, C. P.; Chen, L. H. Biochemistry 1996, 35, 803.
54. Fischer-Valuck, B. W.; Barrilleaux, B. L.; Phinney, D. G.; Russell, K. C.; Prockop, D. J.; O'Connor, K. C. Biotechnol. Lett. 2009.

55. Peng, H.; Yang, Z. K.; Hou, J. Zhonghua Gan Zang Bing Za Zhi 2008, 16, 918.

56. Tanino, Y.; Makita, H.; Miyamoto, K.; Betsuyaku, T.; Ohtsuka, Y.; Nishihira, J.; Nishimura, M. Am. J. Physiol. Lung Cell Mol. Physiol. 2002, 283, L156.

57. Orita, M.; Yamamoto, S.; Katayama, N.; Fujita, S. Curr. Pharm. Des. 2002, 8, 1297.

58. Brown, K. K.; Blaikie, F. H.; Smith, R. A.; Tyndall, J. D.; Lue, H.; Bernhagen, J.; Winterbourn, C. C.; Hampton, M. B. J. Biol. Chem. 2009.

59. Shoichet, B. K. J. Med. Chem. 2006, 49, 7274.

60. Ryckaert, J. P.; Ciccotti, G.; Berendsen, H. J. J. Comput. Phys. 1977, $23,327$.

61. Jain, A. J. Med. Chem. 2003, 46, 499.

62. Stamps, S. L.; Fitzgerald, M. C.; Whitman, C. P. Biochemistry 1998, 37, 10195.

63. Rarey, M.; Wefing, S.; Lengauer, T. J. Comput. Aided Mol. Des. 1996, 10, 41.

64. Clark, R. D.; Strizhev, A.; Leonard, J. M.; Blake, J. F.; Matthew, J. B. J. Mol. Graphics Modell. 2002, 20, 281.

65. Kuntz, I. D.; Blaney, J. M.; Oatley, S. J.; Langridge, R.; Ferrin, T. E. J. Mol. Biol. 1982, 161, 269.

66. Jones, G.; Willett, P.; Glen, R. C.; Leach, A. R.; Taylor, R. J. Mol. Biol. 1997, 267 727.

67. Jones, G.; Willett, P.; Glen, R. J. Mol. Biol. 1995, 245, 43.

68. Muegge, I.; Martin, Y. C.; Hajduk, P. J.; Fesik, S. W. J. Med. Chem. 1999, 42, 2498

69. Eldridge, M. D.; Murray, C. W.; Auton, T. R.; Paolini, G. V.; Mee, R. P. J. Comput. Aided Mol. Des. 1997, 11, 425.

70. Rarey, M.; Kramer, B.; Lengauer, T.; Klebe, G. J. Mol. Biol. 1996, 261, 470. 